\title{
Calculation of Neutral Beam Deposition Accounting for Excited States
}

\author{
T.A. Gianakon
}

10 June 1992

\section{Abstract}

Large-scale neutral-beam auxillary heating of plasmas has led to new plasma operational regimes which are often dominated by fast ions injected via the absorption of an energetic beam of hydrogen neutrals. An accurate simulation of the slowing down and transport of these fast ions requires an intimate knowledge of the hydrogenic neutral deposition on each flux surface of the plasma. As a refinement to the present generation of transport codes, which base their beam deposition on ground-state reaction rates, a new set of routines, based on the excited states of hydrogen, is presented as mechanism for cornputing the attenuation and deposition of a beam of energetic neutrals. Additionally, the numerical formulations for the underlying atomic physics for hydrogen impacting on the constiuent plasma species is developed and compiled as a numerical database. Sample results based on this excited state model are compared with the ground-state model for simple plasma configurations.

\section{Introduction}

As new operational plateaus (H-mode and supershot) have been reached in tokamaks, plasma simulation codes (TRANSP and SNAP) have been required to refine their underlying physics models to accurately reproduce experimental diagnostic measurements. One significant change in these regimes over the previous L-mode and ohmic plasmas has been an increase in the importance of fast ion populations injected via the neutral heating beams. While these fast ion populations may not explain the physics of the transport and confinement of these regimes, an accurate simulation of these regimes cannot be completed without an improved treatment of the spatial absorption of the energetic neutral atoms in the plasma.

Under the present generation of transp ort codes, the neutral beam attenuation is nominally based on simple models that allow only for for ionization or

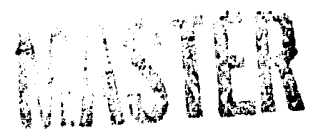


charge exchange events via the ground state of the beam atoms. While such models have advantages in both speed and ease of implementation, recent modelling, which inciudes the effect of the beam excited state populations in the calculation of the beam attenuation, indicates that their inclusion can significantly alter the beam deposition. [1] In the past, such large uncertainities did not alter simulation results, because beam ions represented only a small fraction of the total ion population in the plasma. Furthermore, the computational effort associated with the inclusion of the neutral beam excited state populations represented a sizable increase in code run time, which, when coupled to the small uncertainities, did not strongly motivate their inclusion in the simulations. While this computational effort may still pose a problem, the uncertainities in the beam neutral populations are believed to be no longer acceptable.

To facilitate the inclusion of the beam neutral excited states in the computation of the neutral beam attenuation through the plasina, a new set of codes has been developed. These codes are divided into two packages: 1) a preprocessor package for generating a database of the underlying atomic physics; and 2) a beam-attenuation package for computing beam deposition into ionization and charge exchange channels. Inherent in the des.gn of these codes was the need to insure computational speed, but yet to retain a measure of flexibility. Speed was rec rired to insure routines that would not significantly increase the computational : ad associated with the present generation of transport codes. Flexibility was deemed necessary to handle variations in plasma species mix, species temperature, and different beam energy components, as well as to allow the introduction of updated atomic physics cross-sections. These featcres have been acheived by pre-processing the atomic physics for each plasma species into databases for the reaction rates of interest, i.e., ionization, charge exchange, excitation, de-excitation, and radiative decay. Interpolation within these tables provides a rapid mechanism for computing reaction rates for subsequent use in the numerical solutions of the beam deposition. Further increases in speed are obtained by the use of efficient algorthims for the numerical solution of the coupled, ordinary-differential-equations associated with the beam deposition calculation.

Ultimately, the beam attenuation code returns a "zone-averaged" set of reaction rates that relate the ionization and charge exchange rates for each plasma species to the results of a simple ground state calculation for the zone. This ratio isolates the reaction rates used in this code, so that if alternate reaction rates are used in a different transport code, they may be re-scaled to include this effect.

\section{ATOMIC PHYSICS DATA-BASE}

The atomic physics reaction rates for the beam-deposition routine are generated with two codes for plasma species impact processes, erate.f and prate.f, and one 
code for neutral hydrogen radiative decay, rrate.f. The general philosophy of these codes is that each generates appropriate output files in ASCII format for ionization, charge exchange and excitation/de-excitation events associated with a particuliar plasma species. The rates are computed based on an isotropic, Maxwellian plasma population and a mono-directional, mono-energetic neutral beam population and are given by

$$
\langle\sigma v\rangle=\frac{1}{2} \int_{0}^{\infty} d E_{p} f\left(E_{p}\right) \int_{-1}^{1} d \mu \sigma\left(E_{c}\right)\left(v_{p}^{2}+v_{o}^{2}-2 \mu v_{p} v_{o}\right),
$$

where $f\left(E_{p}\right)$ is a Maxwellian energy distribution for a plasma species at temperature $T(\mathrm{eV})$,

$$
f\left(E_{p}\right)=\frac{2 E_{p}^{1 / 2}}{\pi^{1 / 2} T^{3 / 2}} \exp \left(-E_{p} / T\right)
$$

$\sigma\left(E_{c}\right)$ is the cross-section for the reaction of interest, with $E_{c}$ as the effective collision energy,

$$
E_{c}=E p+\frac{A_{o}}{A_{p}} E_{o}+2 \mu\left(\frac{A_{o} A_{p} E_{p}}{E_{o}}\right)^{1 / 2},
$$

and $\mu$ as the $\cos \theta$ angle in velocity space. The variable $v_{p}$ is the plasma particle velocity at energy $E_{p}$,

$$
v_{p}=\left(\frac{2 E_{p}}{A_{p}}\right)^{1 / 2}
$$

and $v_{0}$ is the velocity of the beam at energy $E_{0}$,

$$
v_{o}=\left(\frac{2 E_{o}}{A_{o}}\right)^{1 / 2}
$$

where $A_{p}$ is the atomic number of the plasma particle and $A_{o}$ is the atomic number of the beam species. All generated rates are in units of $\mathrm{cm}^{3} \mathrm{~s}^{-1}$.

The integration is completed by Laguerre and Gaussian quadratures over the energy and the angular integrals, respectively. The reaction rates are relatively insensitive to the choice of integration order, except for cases when the beam energy and plasma temperatures are comparable. Typically, 16 point Laguerre and 8 point Gaussian quadrature have proven sufficient to generate accurate reaction rates, especially when compared to the inherent uncertainities in the present set of cross-sections. The total number of reaction rates within each file is dictated by the choice of the maximum number of states, nmax, which is determined by the Lorentz ionization limit, memory requirements, and speed. These factors bear directly on the operational apsects of the beam-deposition code and will be discusssed later.

The first file generated by each of the impact proccess codes is comprised of reaction rates for impact ionization events and represents a single vector of 
reaction rates for each quantum state of the beam up to the prescribed maximum state (a total of nmax reaction rates at each beam energy and temperature value in a table). The second file generated (but only for the ion species) is comprised of a vector of charge exchange reaction rates (again, a total of nmax reaction rates per table location). These charge exchange rates do not attempt to track the excited-states of the plasma particle after the charge exchange event, but instead represent the total probability of charge exchange with a plasma species. The final file generated is a matrix which contains rates for excitation and de-excitiation events (a total of $n \max ^{2}$ reaction rates per table location). The diagonal of this matrix is comprised of a sum over excitation events which move particles to states above the prescribed maximum state, and ultimately contribute to the ionization reaction rate. The total number of reaction rates at each beam energy and plasma temperature table location contains nmax $(n \max +2)$ distinct rates.

The first impact process code, erate.f, computes tables of Maxwellian averaged reaction rates, $\langle\sigma v\rangle_{n k l}\left(\mathrm{~cm}^{3} \mathrm{~s}^{-1}\right)$, for electrons interacting with a monoenergetic hydrogen beam. Three subscripts $n, k, l$ refer to reaction rate locations within a table corrsponding to the quantum state of the neutral hydrogen beam, the electron temperature, and the beam energy, respectively. Choices for the electron temperature $T_{k}$ within the table are based on a log spacing between the minimum and maximum table temperatures and are required input values. However, because the electron velocites far exceed the beam velocity, no actual beam energy dependence appears in the calculation of these electron reaction rates. But, for convenience and consistency with other reaction rate tables, the tables for the electron species are dimensioned to have a beam energy dependence. Consequently, all reaction rates within the table are set to zero except for those which correspond to the lowest beam energy component, $l=1$.

The first file erate.f returns contains an array of ionization reaction rates at temperature $T_{k}$ for each quantum state $n$. The analytical form of the crosssections for the electron impact ionization process (and also for the excitation reactions) allows Eq. (1) to be integrated analytically so that the reaction rates are given by [2]

$$
\langle\sigma v\rangle_{n k l}=9.56\left(10^{-6}\right) \frac{\exp \left(-E_{n}\right)}{\left(E_{n}^{2.33}+4.38 E_{n}^{1.72}+1.32 E_{n}\right) T_{k}^{1.5}},
$$

where

$$
E_{n}=\frac{13.598}{T_{k} n^{2}},
$$

and $T_{k}$ is the electron temperature $(\mathrm{eV})$.

The final file generated corresponds to a matrix of reaction rates, $\langle\sigma v\rangle_{m n k l}$, for electron excitation and de-excitation of neutral hydrogen. The additional subscript $m$ refers to an event in which electron impact causes the transition of a beam particle from an initial quantum state given by $m$ to a final quantum 
state given by $\mathrm{n}$. For excitation between the states $1 \mathrm{~s} \rightarrow 2 \mathrm{~s}, 1 \mathrm{~s} \rightarrow 2 \mathrm{p}, 1 \rightarrow 3$, and $2 \rightarrow 3$, the reaction rates are computed via [3]

$$
\langle\sigma v\rangle_{m n k l}=\exp \left\{\sum_{i=1}^{8} A_{i m n}(\log T)^{i}\right\},
$$

where the $A_{\text {imn }}$ are a set of fitting coefficients, which are listed in the subroutine sigvee in Appendix A. While the rates for the reactions $1 s \rightarrow 2 s$ and $1 s \rightarrow 2 p$ are computed, these rates are always combined into a single $1 \rightarrow 2$ rate. All other excitation rates are given by [2]

$$
\langle\sigma v\rangle_{m n k l}=5.19\left(10^{-6}\right) E_{m i}^{1.5} \frac{\exp \left(-E_{m n}\right)}{T E_{n i}^{2.33}}
$$

where

$$
E_{m i}=\frac{13.589}{m^{2} T}
$$

and

$$
E_{n i}=\frac{13.589}{n^{2} T}
$$

and

$$
E_{m n}=E_{m i}-E_{n i} .
$$

The de-excitation rates are computed via the principle of detailed balance which relates a forward reaction to its reverse reaction. Hence, the de-excitation rates are given by [2]

$$
\langle\sigma v\rangle_{m n k l, d e x c}=\langle\sigma v\rangle_{n m k l, e x c}\left(T^{\prime}\right) \frac{T^{\prime} n^{2}}{T m^{2}},
$$

where

$$
T^{\prime}=T-E_{m n},
$$

and $E_{m n}$ is given by Eq. (12).

Finally, the diagnonal elements of this matrix represent excitation events above the maximum table value, nmax, and are given by

$$
\langle\sigma v\rangle_{m n k l}=\sum_{n=n \max +1}^{n m a x+n m o r e}\langle\sigma v\rangle_{m n k l},
$$

where nmore is an input value which dictates the number of extra reaction rates to include in the sum above the maximum value. At a value of nmore $=80$, the summation appears to change by no more than a $0.1 \%$ per additional term. Any additional benefit or accuracy gained by increasing the value of nmore is probably overshadowed by the uncertainities in the cross-sections themselves and represent an unneccessary increase in the computational load. 
The second impact process code, prate.f, produces an array of Maxwellian averaged reaction rates for a hydrogenic $(\mathrm{Z}=1)$ plasma species interacting with a hydrogenic neutral beam. The reaction rates $\left(\mathrm{cm}^{3} \mathrm{~s}^{-1}\right)$ are tabulated versus T/A and $E_{b} / A_{b}$, where $\mathrm{T}$ is the plasma species temperature $(\mathrm{eV}), \mathrm{A}$ is the plasma species atomic number (amu), $E_{b}$ is the beam energy $(\mathrm{eV})$, and $A_{b}$ is the beam atomic number (amu). Choices for $E_{b} / A_{b}$ and T/A are based on a log spacing between the minimum and maximum input values for each parameter. This scaling facilitates the use of a single table for proton, deuterium and tritium plasma and beam species and as will be explained later allows for scaling to impurity species.

The first file this routine creates is a table of reaction rates for the impact ionization of the beam particles. This rate for each quantum state $n$ is computed by substituting into Eq. (1) the expression for the ionization cross-section. The ground-state ionization cross-section is given by [1]

$$
\begin{gathered}
\sigma_{g s, i o n}=1.76\left(10^{-6}\right) n^{4}\left[1.6160 D_{\beta}\left(\frac{1.0999\left(10^{4}\right)}{n^{2} E}\right)+\right. \\
\left.0.2487 D_{\beta}\left(\frac{6.9284\left(10^{3}\right)}{n^{2} E}\right)\right]
\end{gathered}
$$

where $D_{\beta}$ is a tabulated function [4] that is listed in the subroutine dbeta of Appendix B and $E$ is in units of $e V / a m u$. One caveat to the use of this form of the ionization cross-section is the necessity to set to zero any non-physical, negative cross-section values. The ionization cross-sections for the higher excited states are computed based on scaling this ground state cross-section as per the loss cross-section which is discussed below. While such a scaling is valid for regions in which the loss cross-section is strongly dominated by ionization, the validity in the region where charge exchange becomes important has not been proven and is beyond the scope of this report. The ionization cross-section for the higher excited states is then

$$
\sigma_{n, \text { ion }}=\sigma_{g s, \text { ion }} n^{2}\left\{\frac{1-\exp \left[-0.10799\left(10^{-3}\right) n^{-2} E^{-1}\right]}{1-\exp \left[-0.10799\left(10^{-3}\right) E^{-1}\right]}\right\},
$$

where $\mathrm{E}$ is the collision energy in $\mathrm{eV} / \mathrm{amu}$.

The second file generated by prate.f is a table of charge exchange rates. The generation of this table has been hampered by the un-availibilty of a complete set of cross-sections for the full range of quantum states required in the beam deposition code. As a consequence, the computation of charge exchange crosssections are computed by first calculating a loss cross-section which includes both ionization and charge exchange and by then subtracting the ionization cross-section of Eqs. (16) or (17). This loss cross-section from a quantum state $n$ is given by [1]

$$
\sigma_{n, \text { loss }}(E)=1.467\left(10^{-11}\right) n^{2} E^{-1}\left[1-\exp \left(-0.10799\left(10^{-3}\right) n^{2} E\right)\right] .
$$


Thus, the charge exchange cross-section is given by

$$
\sigma_{n, c x}(E)=\sigma_{n, l o s s}(E)-\sigma_{n, i o n}(E)
$$

where if $\sigma_{n, c x}(E)<0$, this cross-section is set to zero.

The final file that prate.f generates is a table of collisional excitation and de-excitation rates. In the interest of brevity and due to the complexity of these excitation cross-sections, the form for these reactions may be found in the subroutine sigpnm of Appendix B.[3], [5] As with the electrons, the deexcitation rates are computed using the principle of detailed balance. Hence, the cross-section for the de-excitaiton reaction $m \rightarrow n$, where $n<m$, is given by

$$
\sigma_{m n k l}(E)=\sigma_{n m k l}\left(E_{m}\right) \frac{n^{2} E_{m}}{m^{2} E},
$$

where

$$
E_{m}=E+E_{n m}
$$

with

$$
E_{n m}=13.598\left(n^{-2}-m^{-2}\right) A^{-1},
$$

and $\mathrm{A}$ is the atomic number of the plasma species. Finally, the diagonal of this matrix represents a summation of reaction rates for excitation events that lead to states not explicitly contained within the table and is given by Eq. (15).

The final set of impact processes concern impurity reaction rates. A separate code has not been provided for these impurity reaction rates, because a reasonable approximation is to simply scale the hydrogenic reaction rates with the $Z$-number of the impurity (i.e., fully stripped He with $Z=2$ ). Such a scaling is possible, because the cross-sections for impurity impact proccesses may be normalized to $\mathrm{Z}[1]$, i.e.,

$$
\sigma\left(\frac{E}{A}\right)=Z \sigma_{z}\left(\frac{E}{A Z}\right) .
$$

Based on this scaling, interpolation within the hydrogenic reaction rate tables is completed based on a temperature of $T /(A Z)$ and a beam energy of $E_{b} /\left(A_{b} Z\right)$. The true reaction rate for the impurity species is then this interpolated value multiplied by $Z^{3 / 2}$. As better cross-sections become available, the routine prate.f could readily be modified to generate a separate set of reaction rates for each of the impurities.

The final atomic physics code, rrate.f, computes a table of radiative deacy rates for the various excited states of neutral hydrogen. No beam energy or plasma temperature dependence occurs in this table. Hence, with a dependence only on an initial and final quantum states, the table contains only $n m a x^{2}$ elements. The radiative decay rates, $A_{m n}\left(s^{-1}\right)$, for the transition $\mathrm{m} \rightarrow \mathrm{n}$ are given by [6]

$$
A_{m n}=8.02862\left(10^{8}\right) f_{m n}\left(n^{-2}-m^{-2}\right) n^{2} m^{-2},
$$


where $f_{n m}$ is the oscillator strength of the transition and is given by [7]

$$
f_{m n}=1.960281 n G\left[m\left(1-n^{2} m^{-2}\right]^{-3},\right.
$$

where $\mathrm{G}$ is a Gaunt factor and is given by [7]

$$
G=G_{o}+G_{1}\left(1-n^{2} m^{-2}\right)^{-1}+G_{2}\left(1-n^{2} m^{-2}\right)^{-2},
$$

with

$$
\begin{gathered}
G_{0}= \begin{cases}1.1330 & , n=1 \\
1.0785 & , n=2 \\
0.9935+0.2328 n^{-1}-0.1296 n^{-2} & , n \geq 3\end{cases} \\
G_{1}= \begin{cases}-0.4059 & , n=1 \\
-0.2319 & , n=2 \\
-0.6282 n^{-1}+0.5598 n^{-2}-0.5299 n^{-3} & , n \geq 3\end{cases}
\end{gathered}
$$

and

$$
G_{2}=\left\{\begin{array}{ll}
0.0714 & , n=1 \\
0.02947 & , n=2 \\
0.3887 n^{-2}-1.181 n^{-3}+1.470 n^{-4} & , n \geq 3
\end{array} .\right.
$$

In summary, three codes must be run prior to use of the beam deposition code: erate.f, prate.f and rrate.f. These routines have been written in FORTRAN, and should be compiled with an appropriate flag set to continue code operation beyond underflow errors. With DEC-FORTRAN this amounts to the inclusion of a -fpe3 as a compiler option. Once these codes have been run, the generated ASCII data files preclude the necessity of re-running the codes again except as revisions are made to the underlying atomic physics.

The total number of reaction rates generated by each program for ntemp temperature locations, nbeam beam locations and nmax quantum states is:

$$
\begin{array}{lc}
\text { Electron Ionization } & n b e a m \cdot n t e m p \cdot n \text { max } \\
\text { Electron Charge Exchange } & 0 \\
\text { Electron Excitation } & n b e a m \cdot n t e m p \cdot n m a x \cdot n \text { max } \\
& \\
\text { Ion Ionization } & n b e a m \cdot n t e m p \cdot n \text { max } \\
\text { Ion Charge Exchange } & n b e a m \cdot n t e m p \cdot n \text { max } \\
\text { Ion Excitation } & n b e a m \cdot n t e m p \cdot n \text { max } \cdot n \text { max } \\
\text { Radiative Decay } & n \text { max } \cdot n \text { max }
\end{array}
$$

The total number of reaction rates is therefore

$$
n \text { total }=n \text { beam } \cdot n t e m p \cdot: \max (3+2 n \max )+n \max ^{2}
$$

with an additional $n$ beam $\cdot n t e m p \cdot n \max (2+n \max )$ rates for each additional impurity reaction rate table not based on the program prate.f. 


\section{DEPOSITION CODE}

The zonal deposition routine, exc_dep, has been developed to compute the transfer of beam particles into plasma ionization and charge exchange channels, but with the inclusion of excited state populations up to either the Lorentz ionization limit or to the maximum number of reaction rates available in the tables. To isolate the under-lying atomic physics, the routine generates a set of "enhancement" factors that represent the ratio of ionization (or charge exchange) based on the excited-state model relative to a similiar calculation based on a ground-state model. The ground-state model, which is commonly used by the present generation of transport codes, assumes that the excited states instanteously decay, so that the dynamics of the beam attenuation depend solely on ground-state ionization and charge exchange reaction rates. By introducing the "enhancement" factors, only minor modification of existing transport codes is required to include this effect in the beam deposition calculation. Additionally, an extensive revamping of the potentially different atomic physics assoriated with a transport code is no longer necessary.

The routine, in the present incarnation, is designed to be used with a neutral particle tracker. The tracker would supply zone-averaged plasma species mix, densities, and temperatures, as well as, beam energy, inital excited state populations, and the zonal width parameters. In this context, the zonal width is the travel distance of the beam across a region (typically between flux surfaces) through which the plasma parameters are deemed constant. The underlying physics is modelled by a convective differential equation for each of the quantum states of the beam, i.e.,

$$
\frac{\partial I_{n}}{\partial t}+v_{b} \frac{\partial I_{n}}{\partial x}=\sum_{m=1}^{n o r d} Q_{n m}^{\prime} I_{m},
$$

where $I_{n}$ is the population intensity of the n-th excited state, $v_{b}$ is the neutral beam velocity, nord is the number of states below the Lorentz ionization limit, and $Q_{n m}^{\prime}$ is the total reaction rate matrix for the zone. However, for beam deposition and attenuation, only the steady-state portion of the convectivedifferential equation need be considered. Therefore, Eq. (31) reduces to the ordinary differential equation

$$
\frac{d I_{n}}{d x}=\sum_{m=1}^{n o r d} Q_{n m} I_{m},
$$


where the matrix $Q_{n m}$ is given by

$$
Q_{n m}=\frac{1}{v_{b}}\left\{\begin{array}{cc}
\sum_{\alpha=1}^{n s p e c} n_{\alpha}\langle\sigma v\rangle_{\alpha m n, e x} & m<n \\
-\left\{\sum_{l=1}^{m-1} A_{m l, r a d}+\sum_{\alpha=1}^{n a p e c} n_{\alpha}\left[\langle\sigma v\rangle_{\alpha m, i o n}+\right.\right. & \\
\left.\left.\langle\sigma v\rangle_{\alpha m, c x}+\sum_{l=1}^{n m a x}\langle\sigma v\rangle_{\alpha m l, e x}\right]\right\} & \\
A_{m n, r a d}+\sum_{\alpha=1}^{n s p e c} n_{\alpha}\langle\sigma v\rangle_{\alpha m n, e x} & m>n
\end{array}\right.
$$

where $A_{m n, r a d}$ are the radiative decay rates, $\langle\sigma v\rangle_{\alpha m n, e x}$ is the Maxwellian averaged reaction rate for impact excitation or de-excitation due to species $\alpha$, $\langle\sigma v\rangle_{\alpha m n, i o n}$ is the Maxwellian averaged reaction rate for impact ionization due to species $\alpha,\langle\sigma v\rangle_{\alpha m n, c x}$ is the Maxwellian averaged reaction rate for charge exchange with species $\alpha, n_{\alpha}$ is the density of species $\alpha$, nspec is the number of plasma species, and nmax is the total number of states in the reaction rate tables (as opposed to nord, the Lorentz limit).

The total number of states, nmax, relates to the total number of quantum states in the atomic physics databases and as such is simply a convenient value to terminate the tables. However, nord is an estimate of the firs quantum state of the neutral beam atoms which resides in the continuum. Excited state populations which fall into this or higher states may be treated as instantly auto-ionized. The value of the Lorentz ionization limit may be estimated by considering excited beam atoms moving at a velocity $\mathbf{v}_{b}$ in a magnetic field $\mathbf{B}$. Such particles are subject to ionization by the Lorentz field $\mathbf{E}_{\mathbf{L}, \mathbf{c}}=\mathbf{v}_{\mathbf{b}} \times \mathbf{B}$. [1]

The critical field at which the ionization becomes unity for the Stark component with the strongest dipole field is given by

$$
E_{L, c}=\frac{\epsilon_{o}}{16 n^{4}},
$$

where $\epsilon_{o}$ is the classical electric field of the nucleus evaluated at the first Bohr radius. Hence, for a given beam velocity and magnetic field, the value of the highest level nord above which levels may be treated $\rightarrow s$ lying in the continuum is

$$
\text { nord }=\frac{1}{2}\left(\frac{\epsilon_{o}}{\left|\mathrm{v}_{\mathrm{b}} \times \mathrm{B}\right|}\right)^{1 / 4} .
$$

The differentiation between nord and nmax is important to compute correctly the ionization rates in the excited state model.

Since the plasma parameters and therefore the reaction rates are constant throughout a zone, the calculation of the "enhancement" factors requires an integration of the population intensities across the zonal width. These integrated 
intensities are folded into the ionization or charge exchange reaction rates to generate total loss rates for each plasma species channel. The loss rate $F_{\alpha}$ for charge exchange is

$$
F_{\alpha, c x}=\sum_{m=1}^{n o r d} n_{\alpha}\langle\sigma v\rangle_{\alpha m, c x} \int_{0}^{w_{x}} d x I_{m}(x),
$$

where $n_{\alpha}$ is the density of the plasma species $\alpha,\langle\sigma v\rangle_{\alpha, m}$ is the charge exchange reaction rate for the plasma species $\alpha, I_{m}(x)$ is the population intensity of the $\mathrm{m}$-th quantum state across the zone, and $w_{z}$ is the travel distance of the beam through the zone. The same general form applies to the calculation of the ionization rate, but the possibility of excitation events above the Lorentz ionization limit must also be included. Hence, the loss rate $F_{\alpha}$ for ionization is

$$
\begin{aligned}
& F_{\alpha, i o n}= \sum_{m=1}^{\text {nord }} n_{\alpha}\left\{\langle\sigma v\rangle_{\alpha m, i o n}+\langle\sigma v\rangle_{\alpha m m, e x}+\right. \\
&\left.\sum_{n=n o r d+1}^{n m a x}\langle\sigma v\rangle_{\alpha m n, e x}\right\} \int_{0}^{w_{z}} d x I_{m}(x),
\end{aligned}
$$

where $\langle\sigma v\rangle_{\alpha m, i o n}$ is the ionization reaction rate for species $\alpha,\langle\sigma v\rangle_{\alpha m m, e x}$ is the ionization reaction rate due to excitation events above the highest state available in the reaction rate tables $(>n \max )$, and $\langle\sigma v\rangle_{\alpha m n, e x}$ is the ionization reaction rate due to excitation events above the Lorentz ionization limit, nord, but less than the maximum table value, nmax. The Lorentz ionization limit also introduces the possibility of a loss channel not due to any plasma species, but due to the auto-ionization of non-zero excited state populations which are above the Lorentz ionization limit as they enter a new zone. This loss rate is dealt with by summing these populations together and storing them as a separate ionization loss rate for the zone.

Finally, to complete the calulculation of the enhancement factor, the loss rate via the ground state model must also be computed and then ratioed to the result of Eq.(35) or (36) as appropriate. The initial vector of excited states $I_{m}(x=0)$, which represents the population intensities of the beam entering the zone, is summed to find the initial beam intensity $I_{o}$, i.e.,

$$
I_{o}=\sum_{m=1}^{\text {nord }} I_{m}(x=0) .
$$

This beam intensity is then used with the toial charge exchange and ionization reaction rate for the ground-state, which is given by

$$
\sigma_{t o t, g s}=\frac{1}{v_{b}} \sum_{\alpha=1}^{n s p e c} n_{\alpha}\left[\langle\sigma v\rangle_{\alpha 1, i o n}+\langle\sigma v\rangle_{\alpha 1, c x}\right],
$$


where $v_{b}$ is the beam velocity, and $\langle\sigma v\rangle_{\alpha 1, i o n}$ and $\langle\sigma v\rangle_{\alpha 1, c x}$ are the ionization and charge exchange rates, respectively, for the collisions of the groundstate of the beam atom with plasma species $\alpha$. This ground-state reaction rate is independent of the Lorentz ionization limit and ignores the effect of all excitation events above the Lorentz limit. The total beam population across the zone is then

$$
I(x)=I_{o} \exp \left(-\sigma_{t o t, g s} x\right) .
$$

Equation (39) is then integrated across the zone and combined with the groundstate charge exchange and ionization reaction rates to generate loss rates for each of the plasma species channels. Therefore, the charge exchange rate with species $\alpha$ is given by

$$
F_{\alpha, c x, g s}=n_{\alpha}\langle\sigma v\rangle_{\alpha 1, c x} \frac{I_{o}}{\sigma_{t o t, g s}}\left[1-\exp \left(-\sigma_{t o t, g s} w_{z}\right)\right],
$$

and the ionization rate is given by

$$
F_{\alpha, i o n, g s}=n_{\alpha}\langle\sigma v\rangle_{\alpha 1, i o n} \frac{I_{o}}{\sigma_{t o t, g s}}\left[1-\exp \left(-\sigma_{t o t, g s} w_{z}\right)\right],
$$

Finally, the enhancement factors are given by

$$
f_{\alpha, i o n}=\frac{F_{\alpha, c x}}{F_{\alpha, c x, g s}},
$$

and

$$
f_{\alpha, c x}=\frac{F_{\alpha, c x}}{F_{\alpha, c x, g n}}
$$

where species $\alpha=0$ of the ionization enhancement factors is reserved for the sum of auto-ionized initial populations that were above the Lorentz limit upon entering the zone.

The flow diagram in Fig. 1 illustrates the operational aspects of the above formalism as implemented in the FORTRAN code exc_dep. As with the atomic physics codes, this routine should be compiled with a flag set which allows program execution to continue beyond underflow errors. Prior to the first call to exc_dep, a call must be made to the routine $Q R E A D$ to initialize and store the reaction rate matrices in the common block /TRRATE/. In addition to initializing /TRRATE/, the variables in the common block/gquad/ should be initialized by setting the zone quadrature order ngmax and by supplying the associated set of Gaussian quadrature ordinates and weights. Once the initialization is complete, the routine exc_dep may be called by specifying the zone plasma conditions via the variables of the common block /SPECI/ and the variables in the declaration of the subroutine. An explanation of each of the above varables and common blocks is provided either in the program file exc_dep of Appendix E or in the include file, exc_dep.com of Appendix F. After 
a succesful calculation, the routine will return a value of $i e r r=0$, the common block /fficx/ will contain the enhancement factors for the zone, and the state intensity vector will contain the excited state populations at the zone edge. Within this common block, the variable ffi $(0)$ is reserved for the instanteous ionization associated with any of the excited state populations above the Lorentz limit.

\section{RESULTS}

Figure 2 illustrates typical ionization enhancement factors for a $60 \mathrm{keV} / \mathrm{amu}$ hydrogen beam impacting normally on a two species plasma composed of electrons and hydrogen. The plasma temperature $\left(T_{e}=T_{i}\right)$ is parabolic with a peak temperature of $10 \mathrm{keV}$ and an edge temperature of $90 \mathrm{eV}$ at a minor radius of $80 \mathrm{~cm}$. Likewise, the density profile $\left(n_{e}=n_{i}\right)$ is parabolic with a centerline density of $10^{14} \mathrm{~cm}^{-3}$ anci an edge density of $10^{13} \mathrm{~cm}^{-3}$. The plasma is divided into $5 \mathrm{~cm}$ radial zones with a uniform magnetic field across the entire plasma. For this particuliar model, the enhancement factors start at $\sim 1$ at the plasma edge and then gradually rise as the beam passes into the plasma core to $\sim 2.2$ for the electron species and $\sim 1.1$ for the proton species. The enhancement factors begin near one because initially only the ground-state of the beam is populated. The increase can then be related to the equilibration of the groundstate with the excited states and the larger ionization reaction rates associated with these excited states. The large enhancement factor $(\sim 2.2)$ for the electron species should not be construed as a radical change in the overall ionization rate within the zone, because the electron impact ionization is normally small except for regions near the plasma edge. However, the enhancement factor for the proton species, which is much smaller $(\sim 1.1)$ can significantly affect the deposition profile since the overall ionization is strongly dominated by ion impact proccesses.

Figure 2 also addresses the sensitivity of the enhancement factor to the Lorentz ionization limit and hence the magnitude of the magnetic field. In particuliar, the magnetic field has been adjusted to $6,0.5$, and $0.01 \mathrm{~T}$ such that the number of excited states, nord, takes on values of 5,10 , and 15 , respectively. As the number of excited states is increased, the magnitude of the enhancement factor for all the zones increases for the ion species but decreases for the electron species. Several effects interplay with each other to produce these trends. First, excitation events that previously led to states above the Lorentz limit are no longer ionization events and will therefore no longer contribute to the ionization enhancement factor. Second, the ionization rates for these higher excited states are much stronger than any of the preceeding lower excited states and will therefore increase the ionization when these states are strongly populated. Finally, increases in the overall attenuation of the beam populations may lead to decreases in those enhancement factors that contribute weakly to the overall 
attenuation of the beam. With these observations, the increase in the ion ionization enhancement facto: relates to the strong excitation of beam populations to the higher excited states and their subsequent rapid ionization. By a similiar argument, the enhancement factor for the electron species is expected tc also exhibit a comparable increase. However, since the beam attenuation is strongly dominated by ion impact processes, the large increase in the ion enhancement factor is indicative of an overall decline in the beam populations. This decline in beam populations manifests itself as a lower enhancement factor for the electron species.

Figure 3 illustrates typical ionization enhancement factors for the previously described plasma and beam conditions, but with the added complication of a helium species with $Z_{\text {eff }}=1.5$. As with the previously described problem, the enhancement factor for all three species begins near one at the plasma edge and then gradually rises as the beam moves into the core regions. Likewise, decreases in the magnetic field cause an increase in the ionization enhancement factor for both the hydrogen and helium species, but a decrease in the enhancement factor for the electron species. Surprisingly, the enhancement factors for hydrogen and helium do not deviate from the same value until the magnetic field becomes relatively small. Additionally, the introduction of the new species causes a moderate decrease in the ionization enhancement factor for both the hydrogen and for the electron species. This decline is related to the additional attenuation of the beam populations caused by the helium species.

A significant complication of adding the excited states into a beam deposition model is potential for a sizable increase in the computational time. Table I illustrates typical timing information based on the previously described plasma and beam conditions. As expected, the run time appears to increase in a nearly linear manner as the number of species is increased. However, the run time appears to increase cubicly with the number of excited states nord. Consequently, for a true heating beam which passes through edge. zones with a strong perpendiculiar magnetic field and through core zones with a weak perpendiculiar magnetic field, the actual computation time is a sum over these times weighted for zone width. Furthermore, since the generated deposition represents only a single beam line, the increase in time will directly relate to the total number of beam lines used in any associated transport code.

\section{Acknowledgements}

I would like to express my gratitude for assistance from Doug McCune and Mike Zarnstorff at PPPL for discussions of relevant features that were sought in a beam deposition code and would like to especially thank Ray Fonck for the numerous discussions on atomic physics issues.

I would also like to thank Jim Callen for financial support via US DoE grant number DE-FG02-86ER53218. 


\section{References}

[1] R.K. Jannev, C.D. Boley, D.E. Post, Nuciear Fusion, Vol. 29, NO. 12 (1989) 2125.

[2] L. Vriens and A.H.M. Smeets, Physical Review A, Vol. 22, NO. 3 (1980) 940 .

[3] Janev PPL-TM-368

[4] R.K. Janev and L.P. Presnyakov, J. Phys. B. Atom. Molec. Phys. Vol. 13 (1980) 4233.

[5] J.G. Lodge, I.C. Percival, and D. Richards, J. Physics B: Atom. Molec. Phys., Vol 9, No. 2 (1976) 239.

[6] W.L. Wiese and G.A. Martin, Wavelengths and Transition Probabilities for Atoms and Atomic Ions, Wash. D.C.: U.S. Dept of Commerce, N.B.S., U.S., G.P.O., (1980) 359.

[7] L.C. Johnson, Astrophysical Journal, Vol 174, (1972) 227. 
TABLE I. Average user time per $5 \mathrm{~cm}$ zone versus the perpendicular magnetic field and the number of plasma species in the zone. User time refers to the output from the time command during program execution on a DECstation 5000 under Ultrix 4.2. The test program was compiled with full optimization with DEC Fortran for ULTRIX RISC Systems Version 3.1.

\begin{tabular}{|c|r||r|r|r|}
\hline \multicolumn{2}{|c|}{} & \multicolumn{3}{|c|}{$\begin{array}{c}\text { Average System Time per Zone } \\
(\mathrm{s})\end{array}$} \\
\hline $\begin{array}{c}B_{\text {perp }} \\
(\mathrm{T})\end{array}$ & nord & 2 species & 3 species & 4 species \\
\hline 6 & 5 & 0.16 & 0.17 & 0.18 \\
0.5 & 10 & 0.39 & 0.48 & 0.56 \\
0.01 & 15 & 1.98 & 2.58 & 3.04 \\
\hline
\end{tabular}




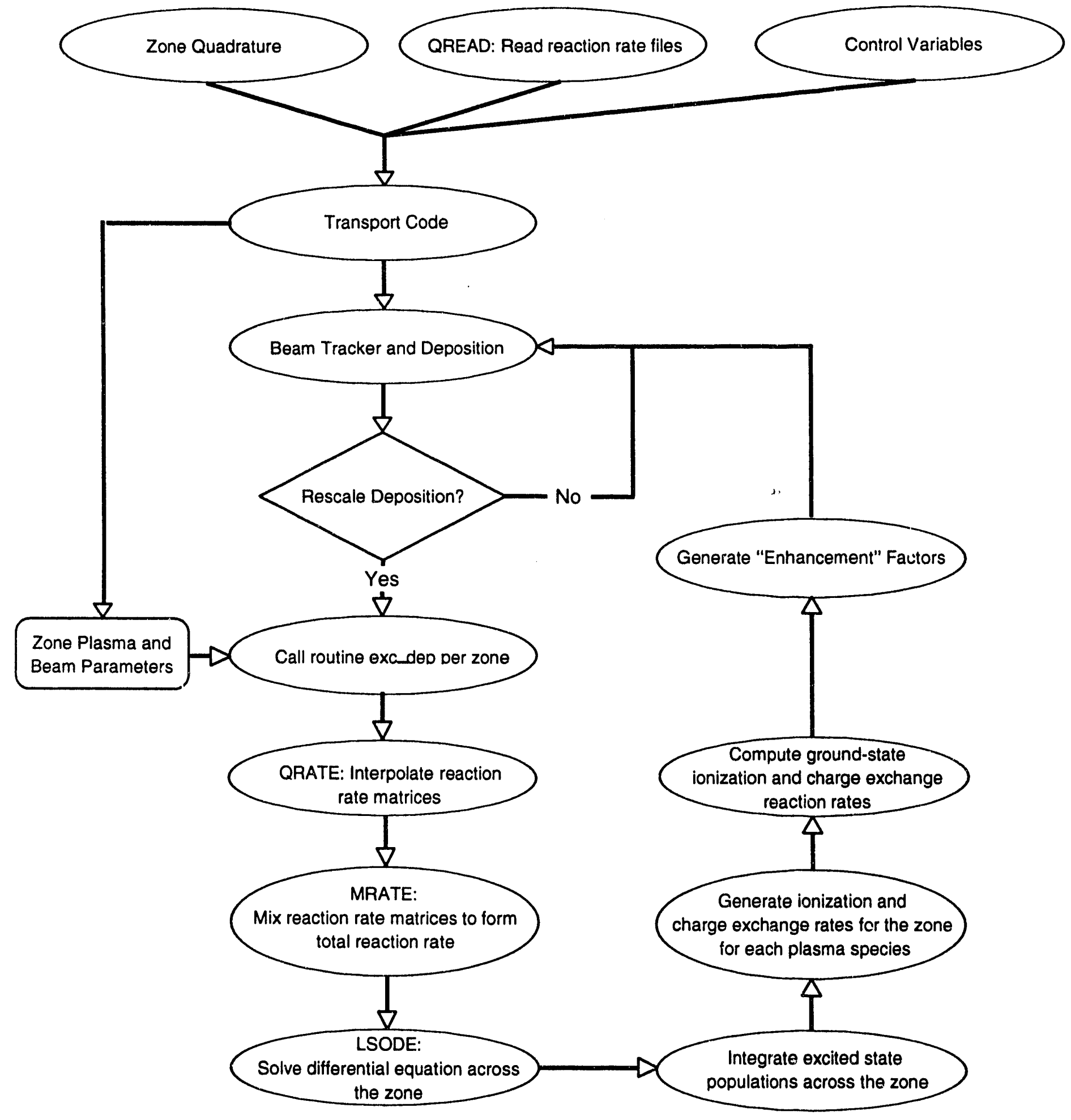

FIGURE 1. Flow diagram of the routine exc_dep with a connection to a neutral beam particle tracker and associated transport code. The routine requires zone quadrature data and the reaction rate matrices to be passed via the transport code. Each call to the routine computes the enhancement factor for a single zone with associated beam and plasma conditions. 


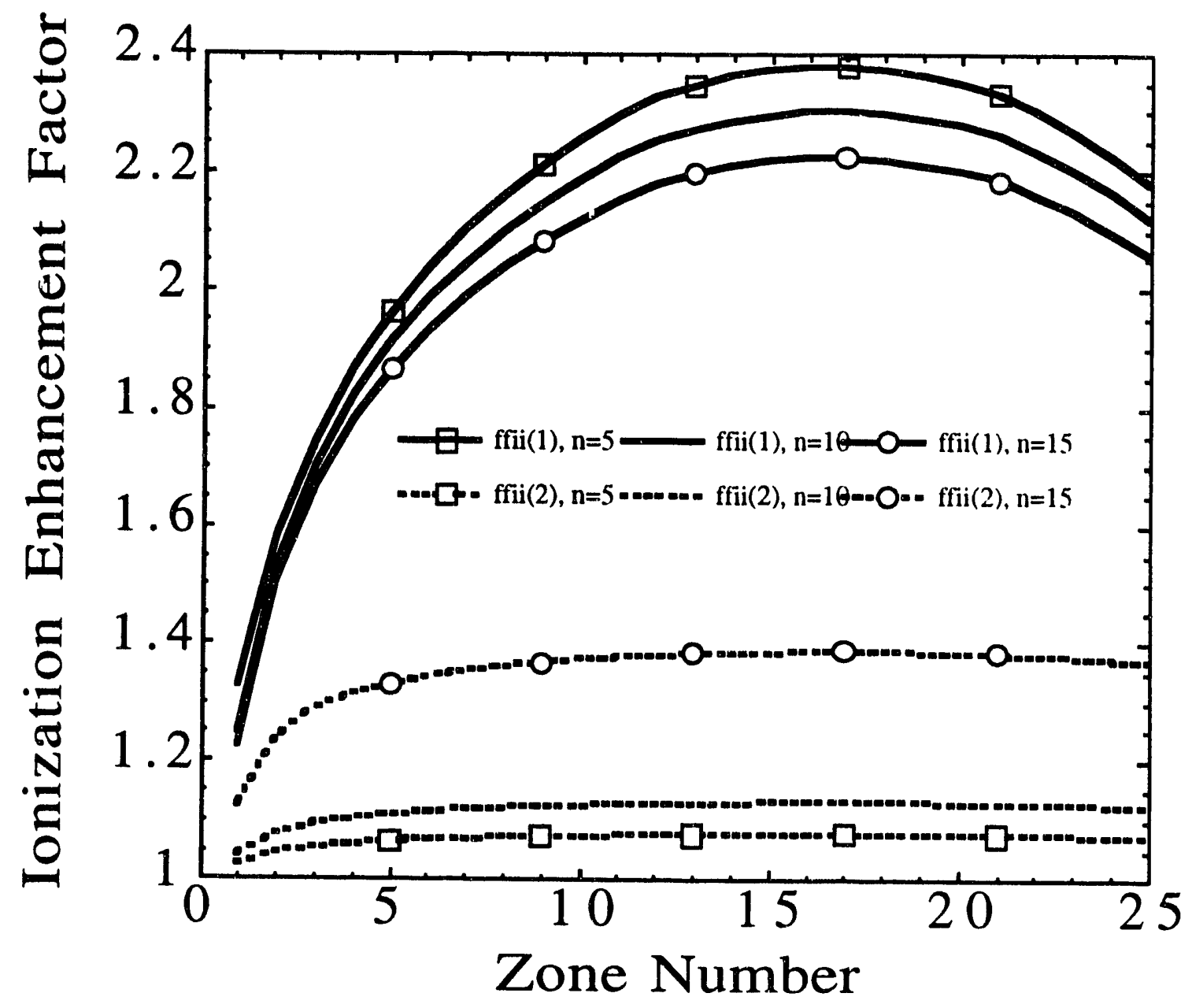

FIGURE 2. Impact ionization scale factor for a $60 \mathrm{keV} / \mathrm{amu}$ hydrogen beam impinging on a two species plasma composed of electrons and hydrogen. The plasma has a minor radius of $80 \mathrm{~cm}$ and each zone is $5 \mathrm{~cm}$ in width. The temperature profile is parabolic with a peak temperature of $10 \mathrm{keV}$ and an edge temperature of $90 \mathrm{eV}$. The number $\mathrm{n}$ refers to the Lorentz ionization limit and is the total number of equations retained in the calculation. 


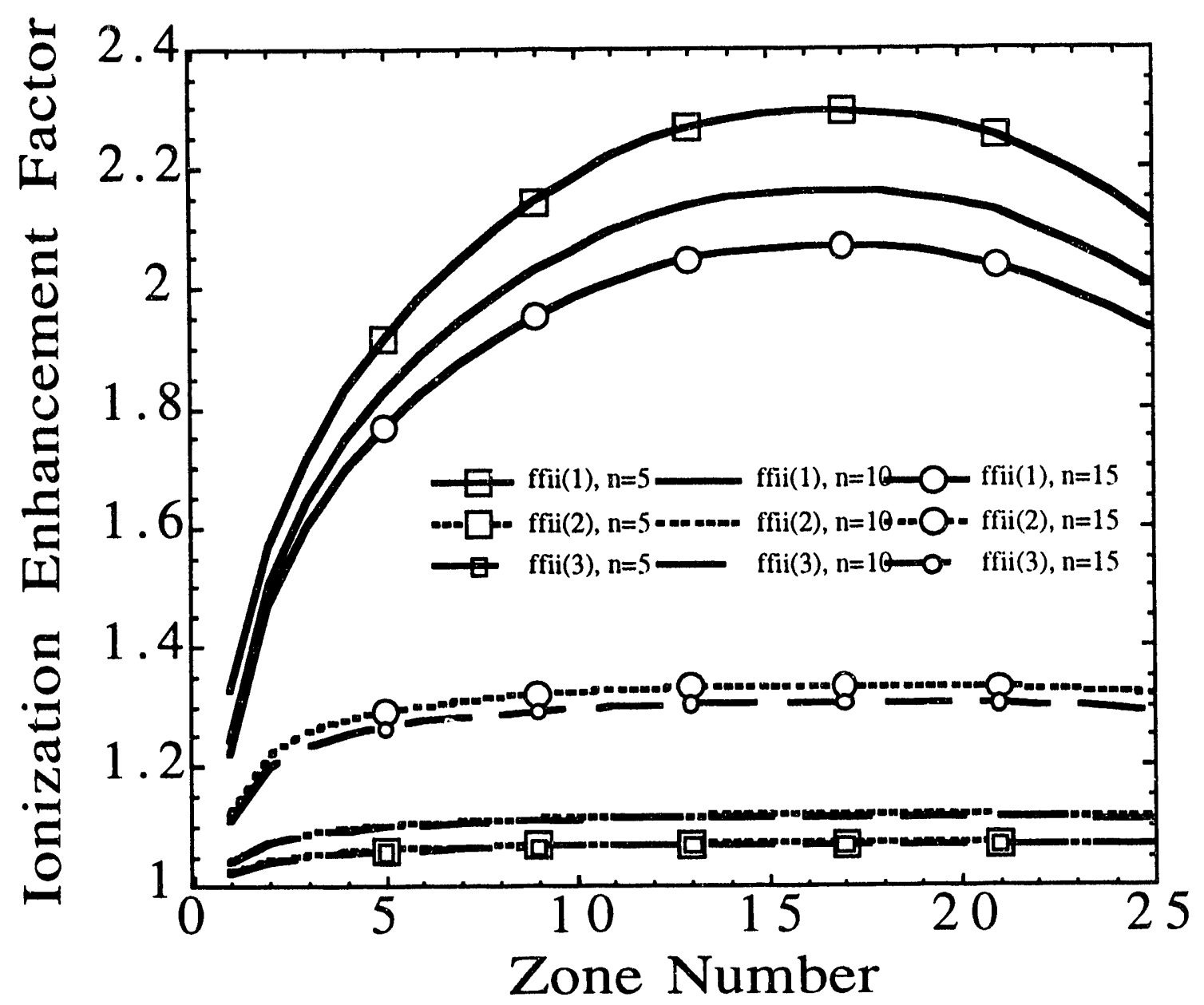

FIGURE 3. Impact ionization scale factor for a $60 \mathrm{keV} / \mathrm{amu}$ hydrogen beam impinging on a three species plasma composed of electrons, hydrogen, and helium. The plasma has a Zeff:1.5 over the entire plasma. The plasma has a minor radius of $80 \mathrm{~cm}$ and each zone is $5 \mathrm{~cm}$ in width. The temperature profile is parabolic with a peak temperature of $10 \mathrm{keV}$ and an edge temperature of $90 \mathrm{eV}$. The number $n$ refers to the Lorentz ionization limit and is the total number of equations retained in the calculation. 


\section{A Electron Reaction Rate Code erate.f}

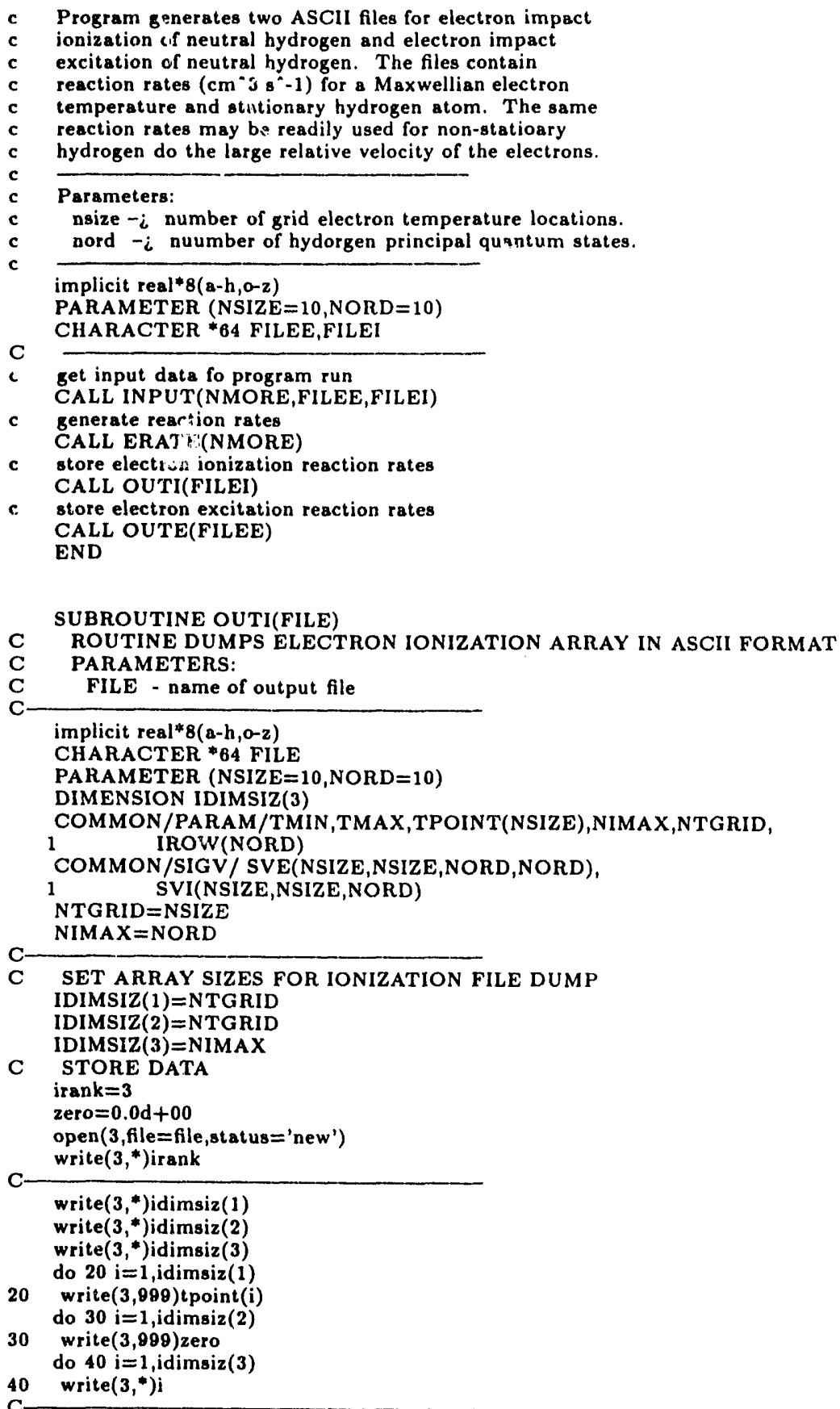

C SET ARRAY SIZES FOR IONIZATION FILE DUMP $\operatorname{IDIMSIZ}(1)=N T G R I D$ IDIMSIZ(2)=NTGRID $\operatorname{IDIMSIZ}(3)=$ NIMAX

C STORE DATA

irank $=3$

zero $=0.0 \mathrm{~d}+00$ open (3,file=file,status ='new') write $\left(3,{ }^{*}\right)$ irank

Cwrite $(3, *)$ idimsiz(2) write( $3, *)$ idimsiz $(3)$ do $20 \mathrm{i}=1$, idimsiz(1)

20 write $(3,999)$ tpoint(i) do $30 \mathrm{i}=1$,idimsiz(2)

30 write $(3,999)$ zero do $40 \mathrm{i}=1$, idimsiz(3)

40 write $(3, *) \mathrm{i}$ 


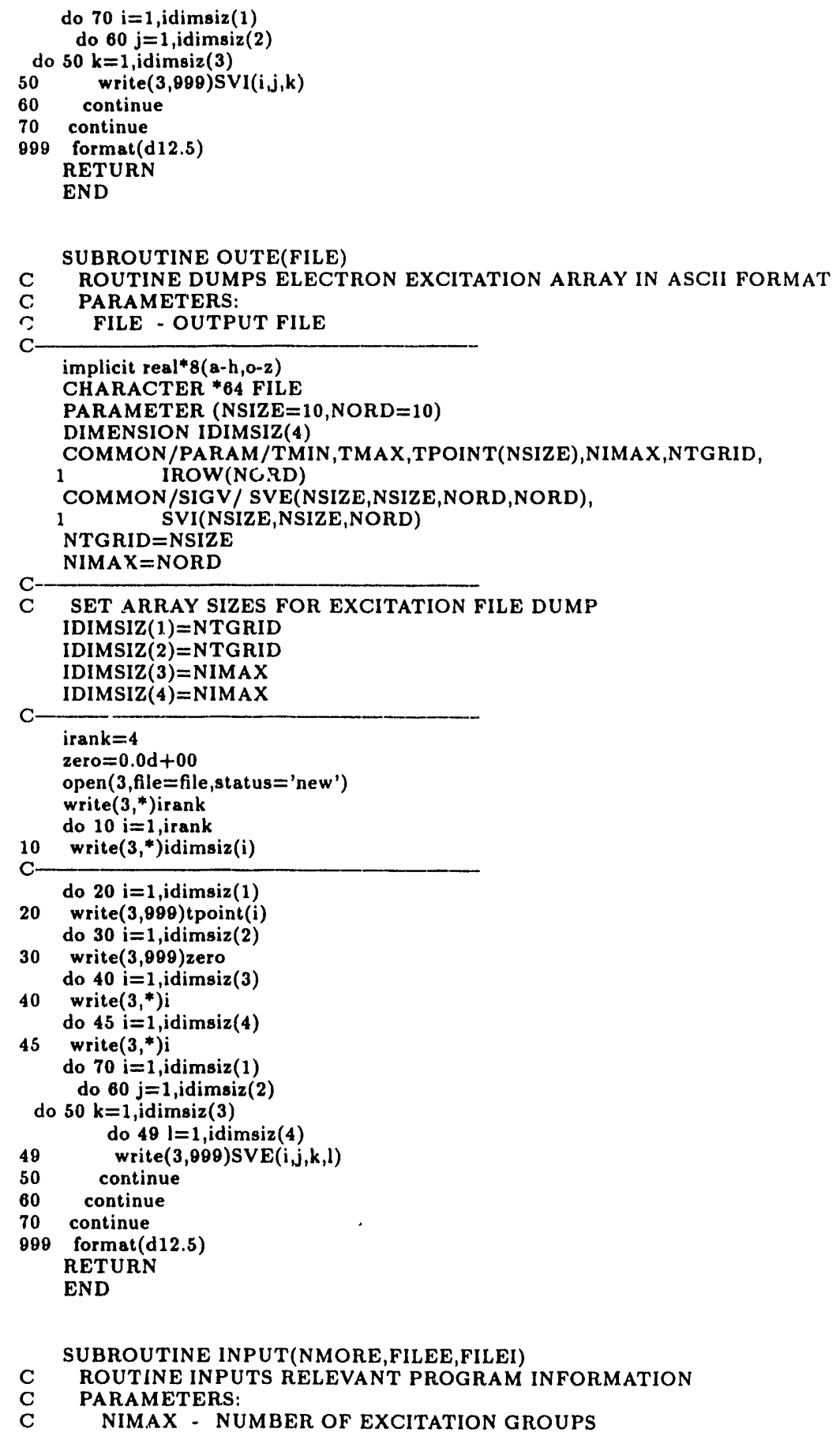




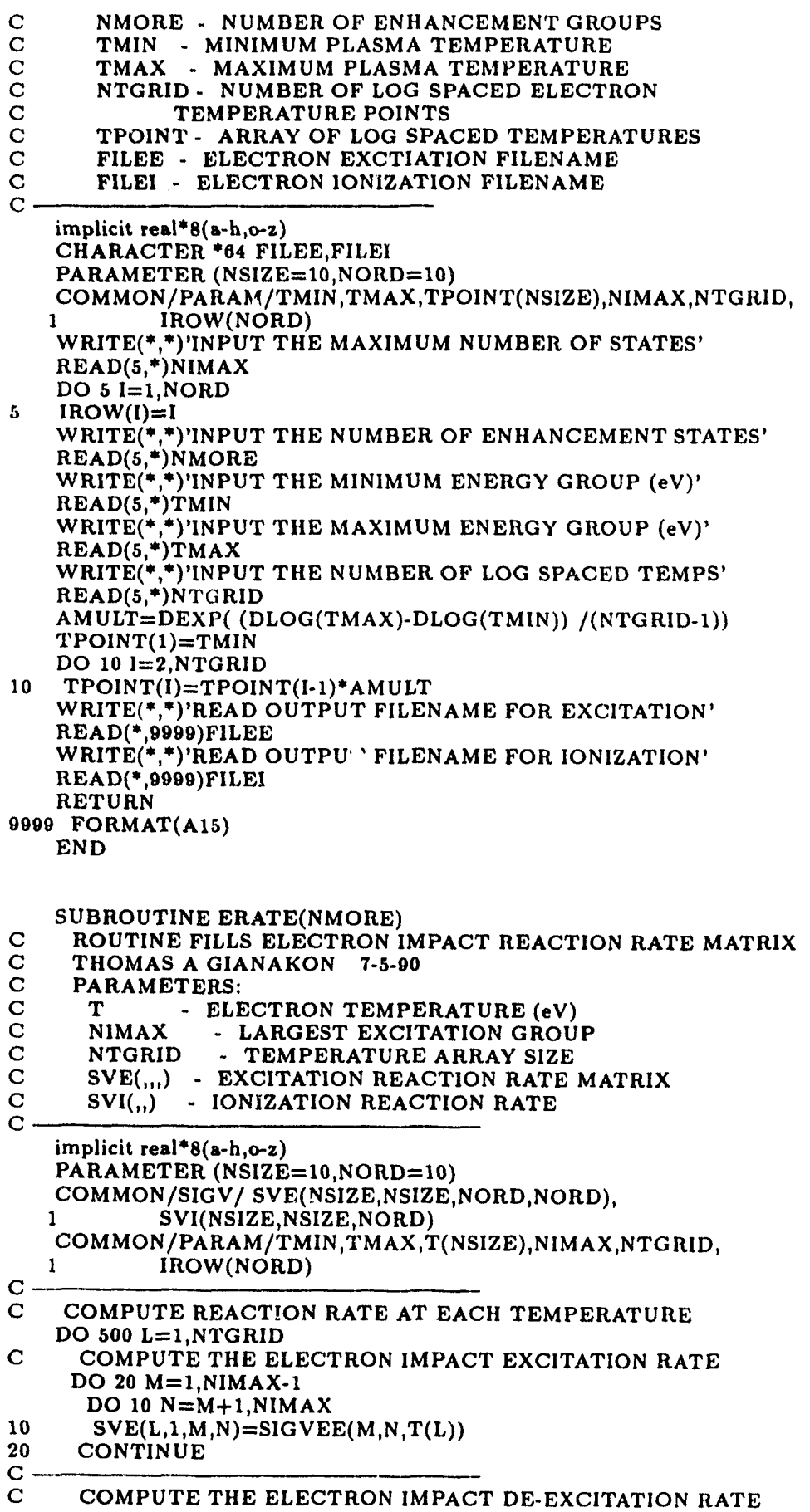




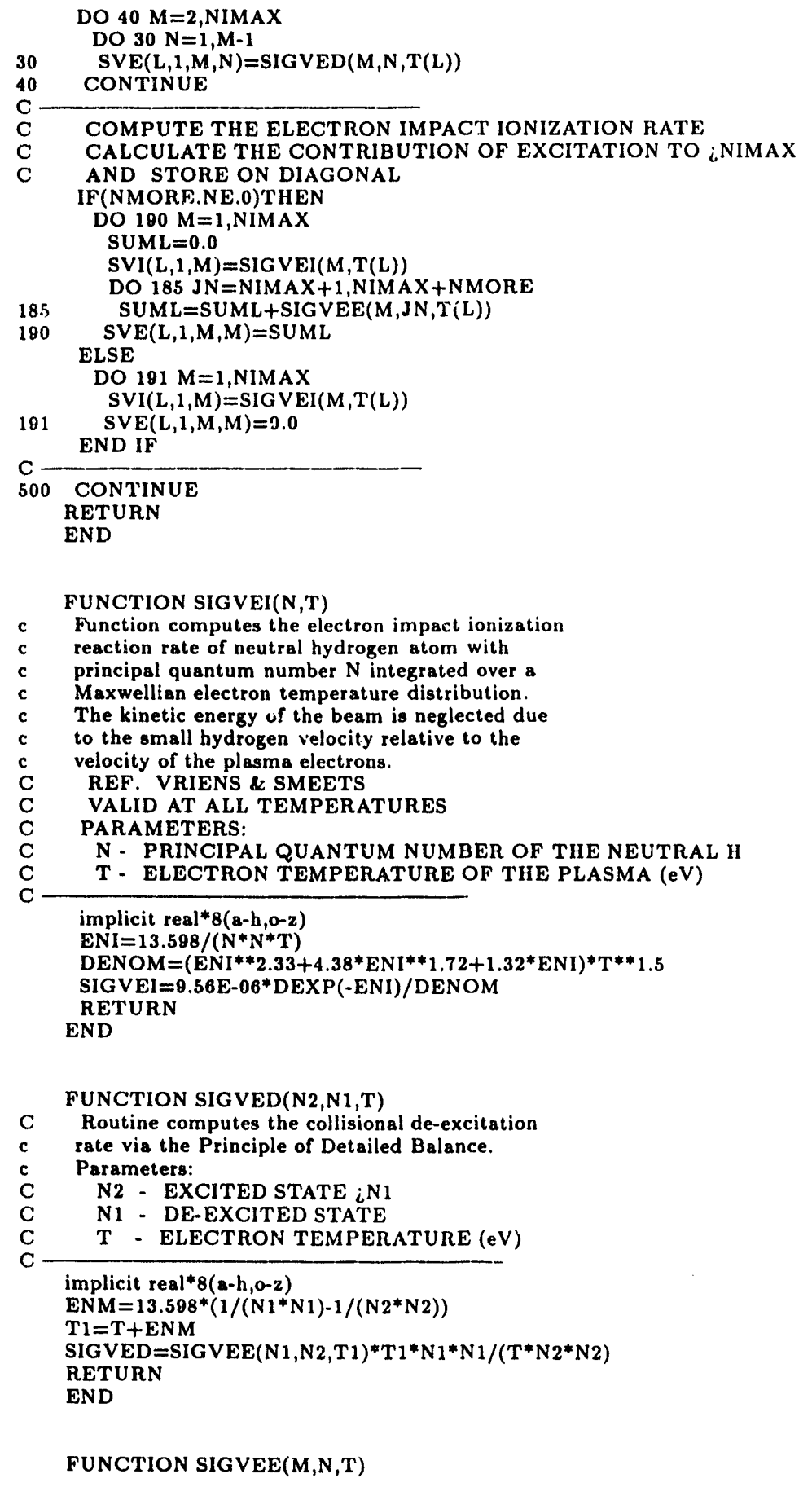




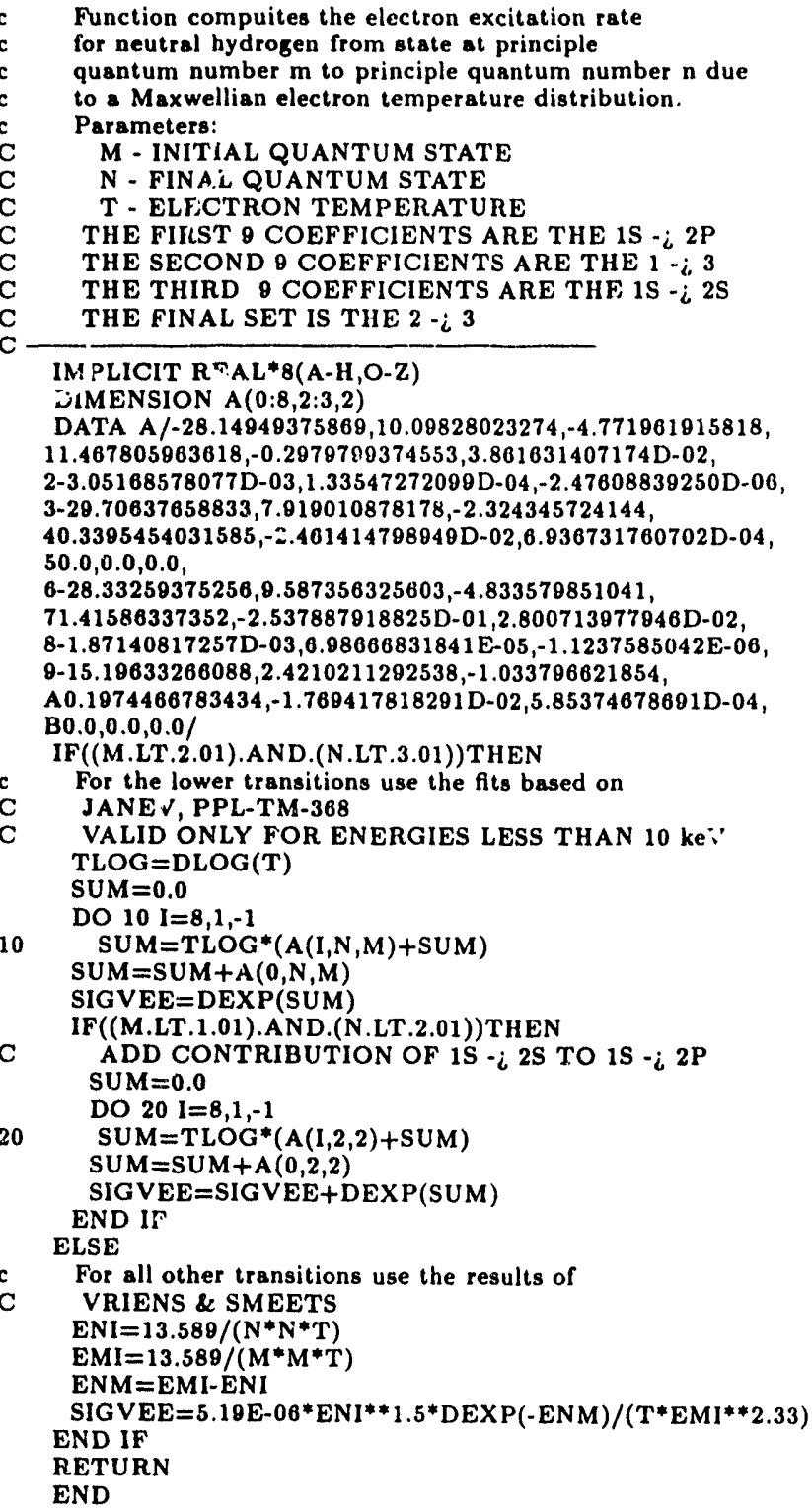




\section{B Proton Reaction Rate Code prate.f}

c Program generates three ASCII files for the proton

c impact ionization, charge exchange, and excitation

c of a neutral hydrogen beam. The files contain reaction

c rates rates $\left(\mathrm{cm}^{\wedge} 3 \mathrm{~s}^{\wedge}-1\right)$ for an isotropic, Maxwellian

c proton ditribution and a mono-directrional, mono-

c energetic hydrogen beam. These rates readily scale

c to impurity reactions with normalized temperature $\mathrm{T} / \mathrm{AZ}$

c and energies $E / A Z$ and a scale factor of $Z^{*}(-3 / 2)$.

Parameters:

c Parameters:

c nord $-i$ number of principle quantum states for

c hydrogen to compute rates for

c Compilation:

c Requires linking with a library to compute Gaussian

c and Laguerre quadrature. The user will be prompted

c for other relevant data.

implicit real*8(a-h,o-z)

PARAMETER (NSIZE $=10$, NORD $=15$ )

CHARACTER *64 FILEE,FILEI,FILEC

C

c get input dats for program run

CALL INPUT(NMORE,FILEE,FILEI,FILEC)

c generate rates

CALL PRATE(NMORE)

C DUMP DATA

CALL OUTI(FILEI)

CALL OUTC(FILEC)

CALL OUTE(FILEE)

END

SUBROUTINE OUTI(FILE)

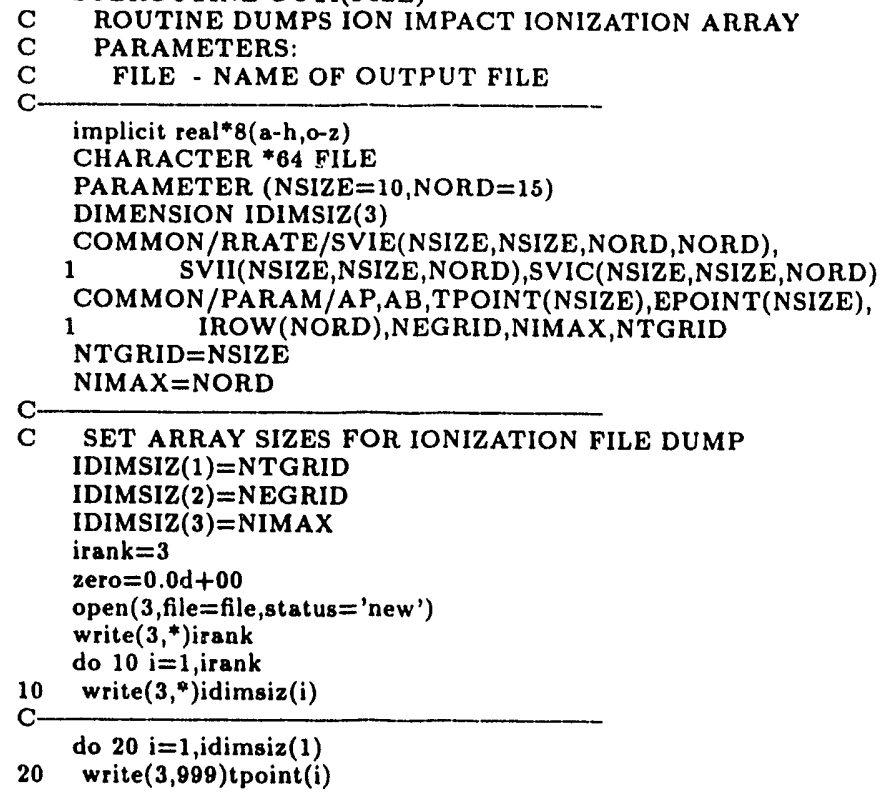




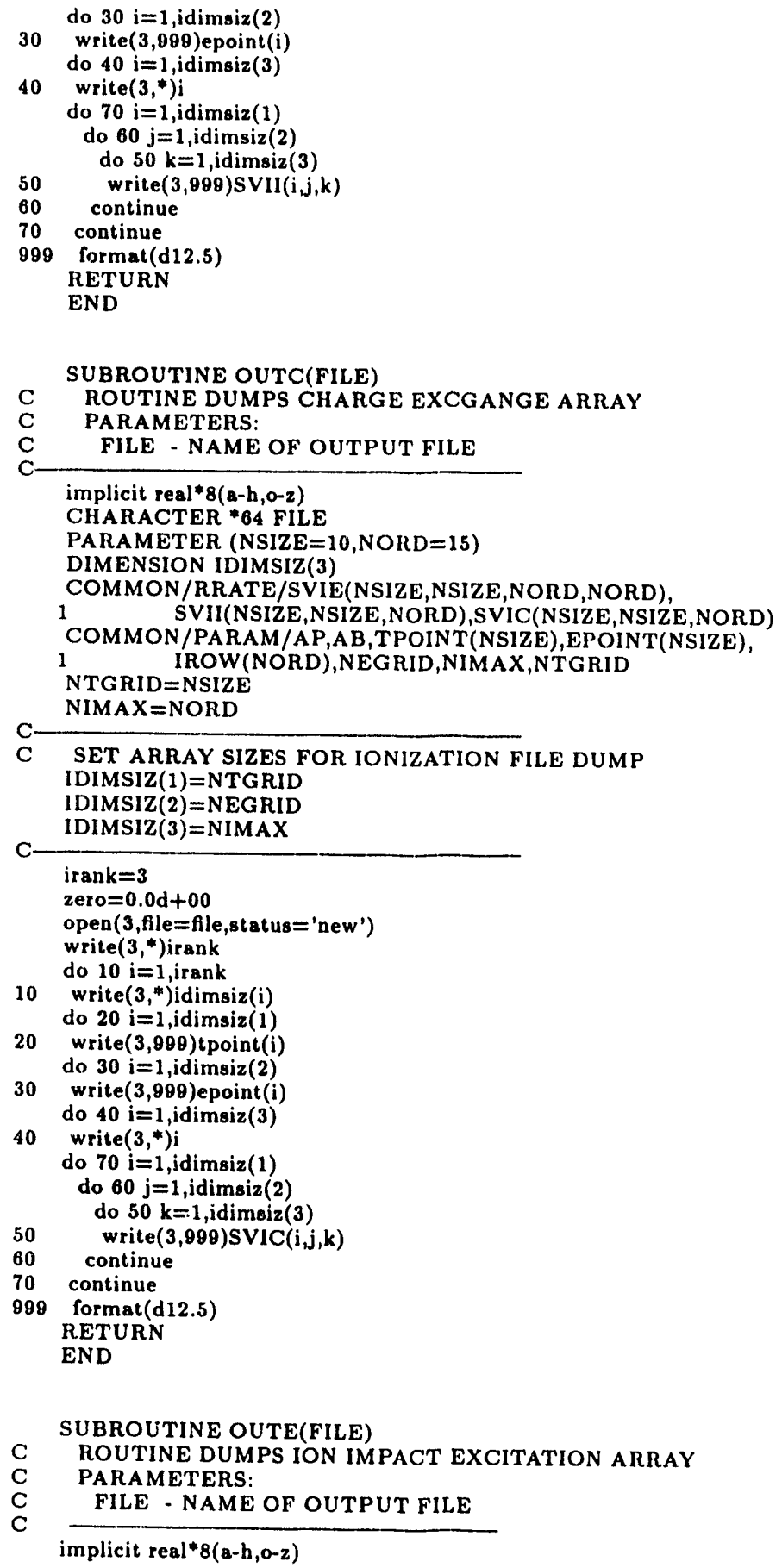




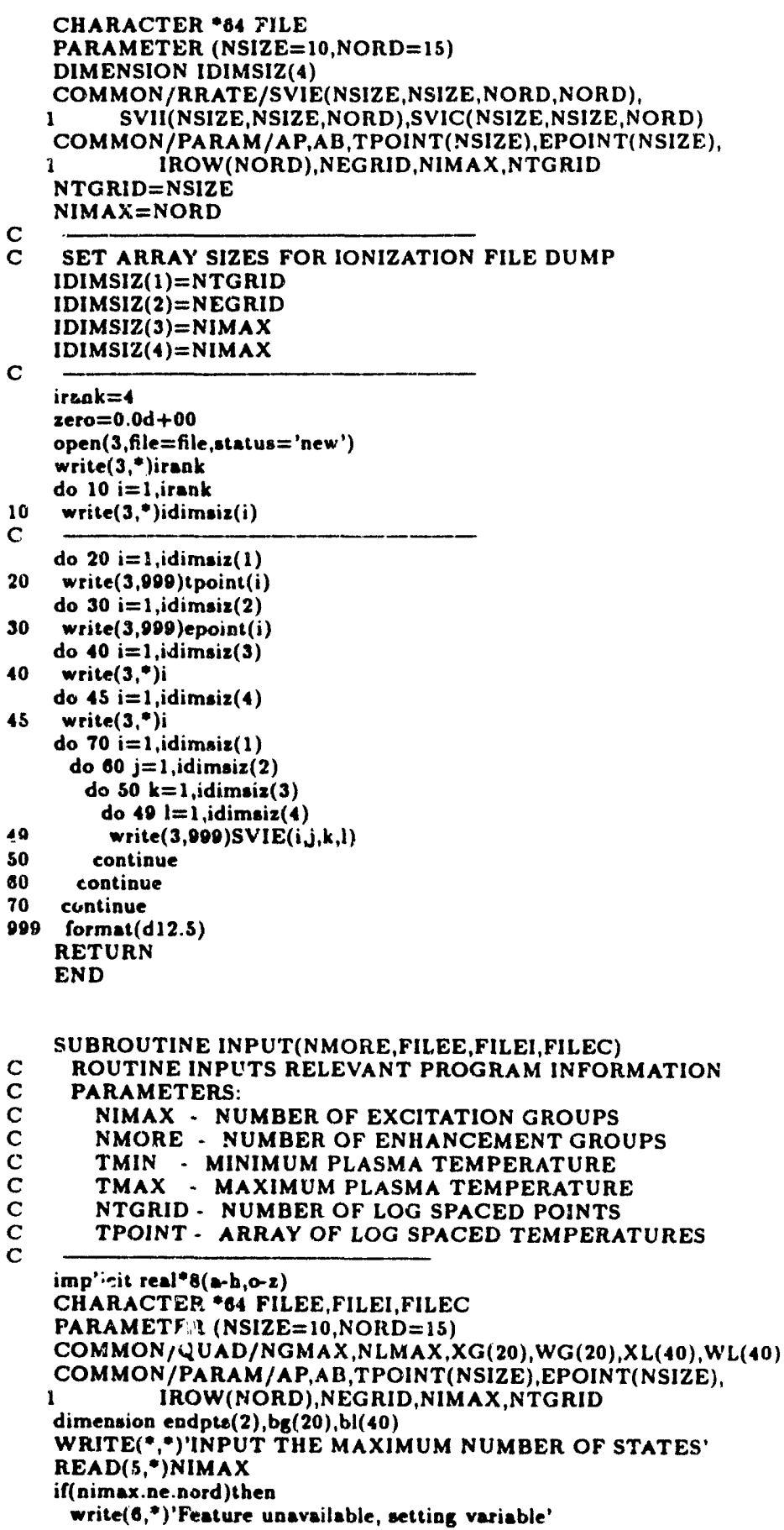


write $(6, *)$ 'equal to nord.'

nimax $=$ nord

end if

DO $5 \mathrm{I}=1$, NIMAX

$5 \quad \operatorname{IROW}(1)=$

WRITE(*, 'INPUT THE NUMBER OF ENHANCEMENT STATES'

READ $(5, *)$ NMORE

WRITE(**)'INPUT THE MINIMUM TEMPERATURE (eV)'

READ(5,*)TMIN

WRITE(*,*)'INPUT THE MAXIMUM TEMPERATURE (eV)'

READ (5,*)TMAX

WRITE(*,*)'INPUT THE NUMBER OF LOG SPACED TEMPS'

READ $(5, *)$ NTGRID

WRITE(**)'INPUT THE PLASMA ATOMIC NUMBER

READ (5,*)AP

AMULT $=$ DEXP( (DLOG $($ TMAX)-DLOG(TMIN $)) /($ NTGRID-1))

TPOINT $(1)=T M I N$

DO $10 \mathrm{I}=2$,NTGRID

10 TPOINT $(I)=$ TPOINT $(1-1) * A M U L T$

WRITE(*,*)'INPUT THE MINIMUM BEAM ENERGY (eV)'

READ $(5, *)$ EMIN

WRITE(*,*)'INPUT THE MAXIMUM BEAM ENERGY (eV)'

READ $(5, *)$ EMAX

WRITE(*,*)'INPUT THE NUMBER OF LOG SPACED BEAMS'

READ $(5, *)$ NEGRID

WRITE $(*, *)$ 'INPUT THE BEAM ATOMIC NUMBER'

READ(5,*)AB

AMULT =DEXP( (DLOG(EMAX)-DLOG(EMIN)) /(NEGRID-1))

EPOINT(1) $=$ EMIN

DO $20 I=2, N E G R I D$

20 EPOINT $(I)=$ EPOINT $(I-1)^{*}$ AMULT

C INTEGRATION COEFFICIENTS \& WEIGHTS

WRITE(*,*)'INPUT GAUSSIAN INTEGRATION

READ(5,*)NGMAX

call gaussq $(1, \mathrm{ngmax}, 0.0,0.0,0$,endpts, bg, $x g, w g)$

WRITE(*, )'INPUT LAGUERRE INTEGRATION ORDER'

READ $(5, *)$ NLMAX

(6, nlmsx, $0.0,0.0,0$, endpts, bl, xl,wl)

WRITE(*,*)'READ OUTPUT FILENAME FOR EXCITATION'

READ(*,0090)FILEE

WRITE(*,")'READ OUTPUT FILENAME FOR IONIZATION'

READ $(*, 0999)$ FILE

WRITE(*,')'READ OUTPUT FILENAME FOR CHARGE EXCHANGE'

READ $(*, 0099)$ FILEC

RETURN

9990 FORMAT(A64)

END

SUBROUTINE PRATE(NMORE)

C ROUTINE COMPUTES THE REACTION RATE MATRIX FOR

C NEUTRAL HYIROGEN IMPACTING ON A HYDROGEN PLASMA

C THOMAS A GIANAKON 7-16-90

C PARAMETERS:

TEMP - ION TEMP FOR ISOTROPIC MAXWELLIAN (eV)

AP - ATOMIC MASS OF PLASMA PARTICLE (AMU)

EB - BEAM ENERGY (eV)

AB - ATOMIC MASS OF BEAM PARTICLE (AMU)

$Q(N, M)$ - REACTION RATE MATRIX

NIMAX - MAXIMUM EXCITATION STATE

NGMAX - GAUSSIAN INTEGRATION ORDER 


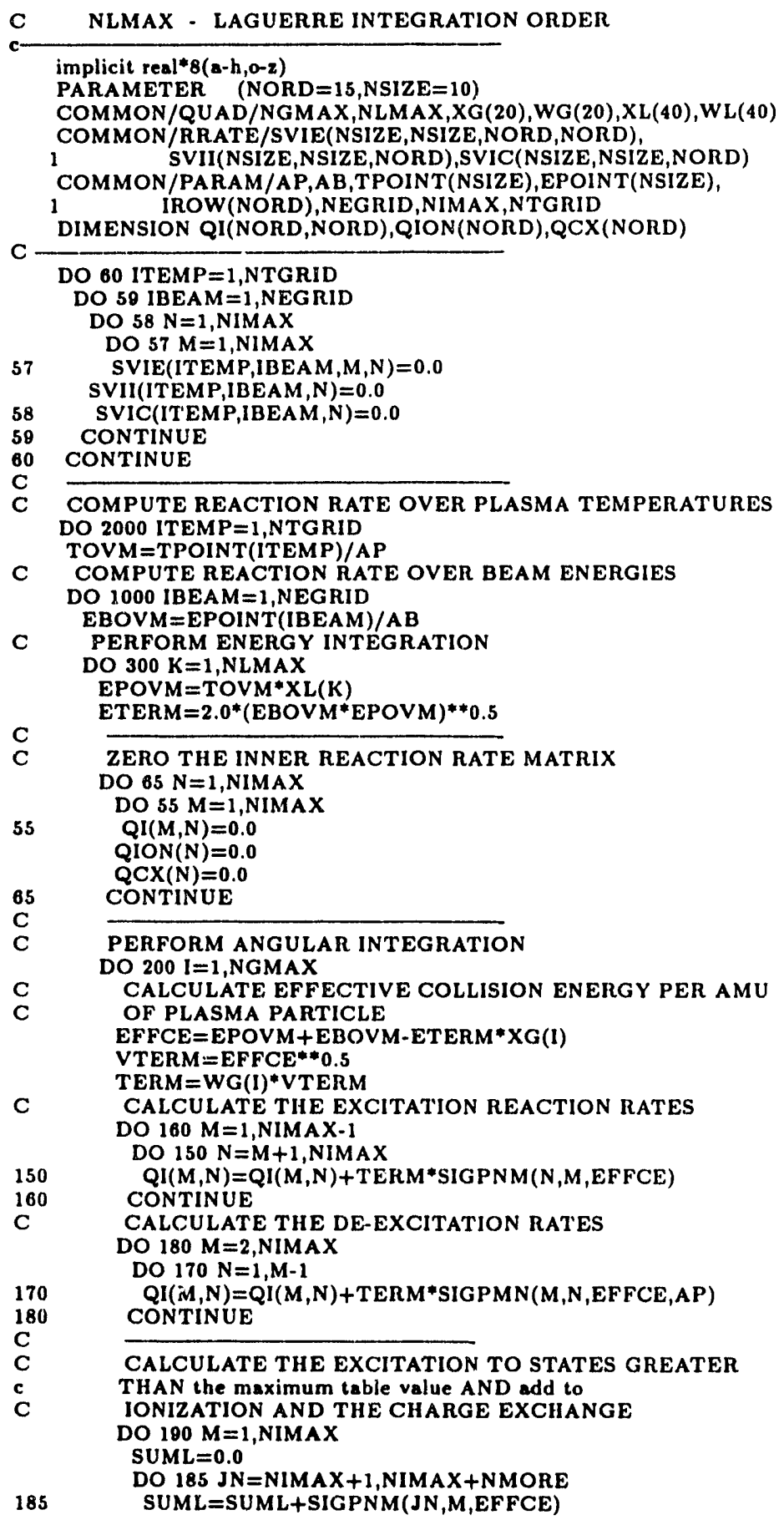




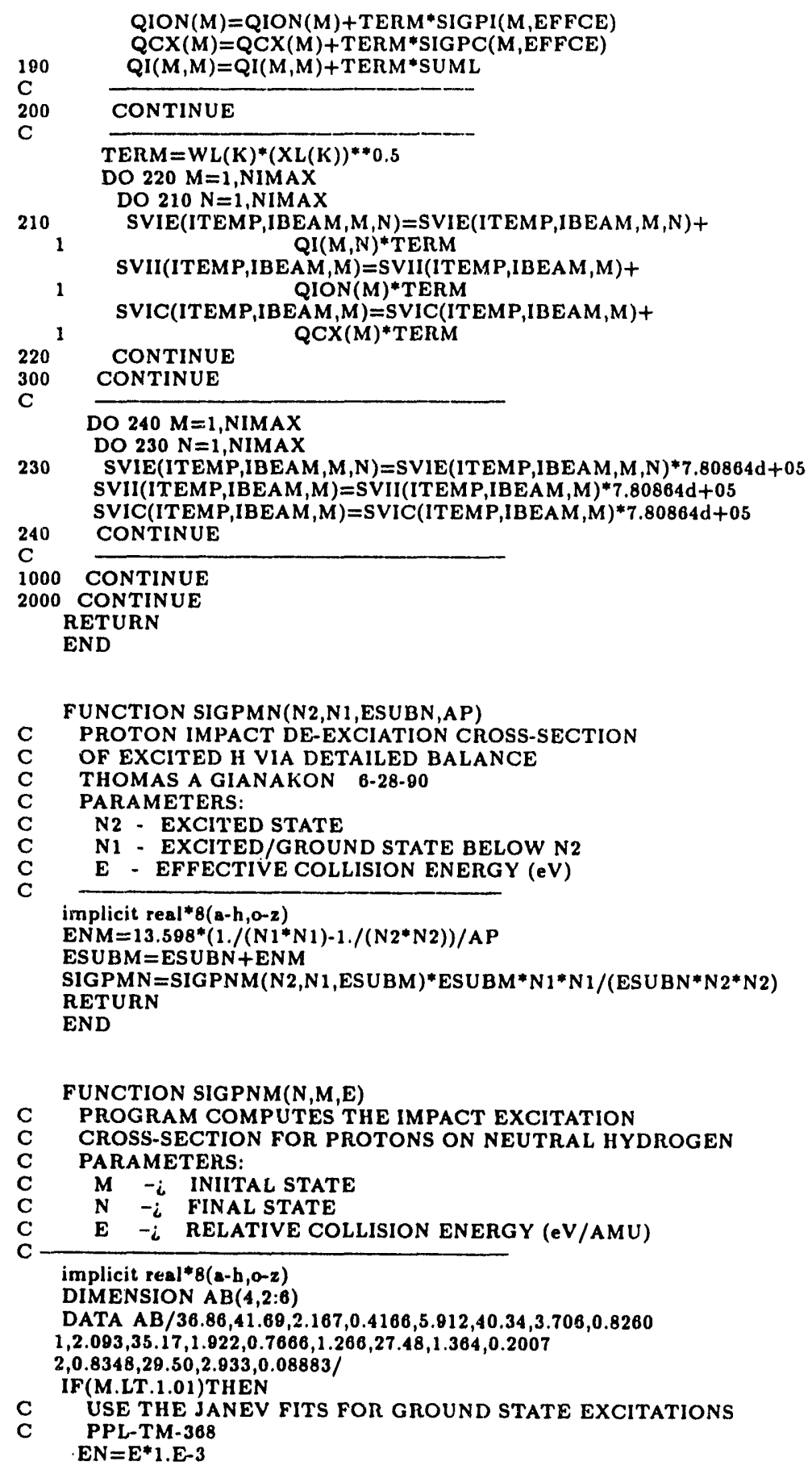




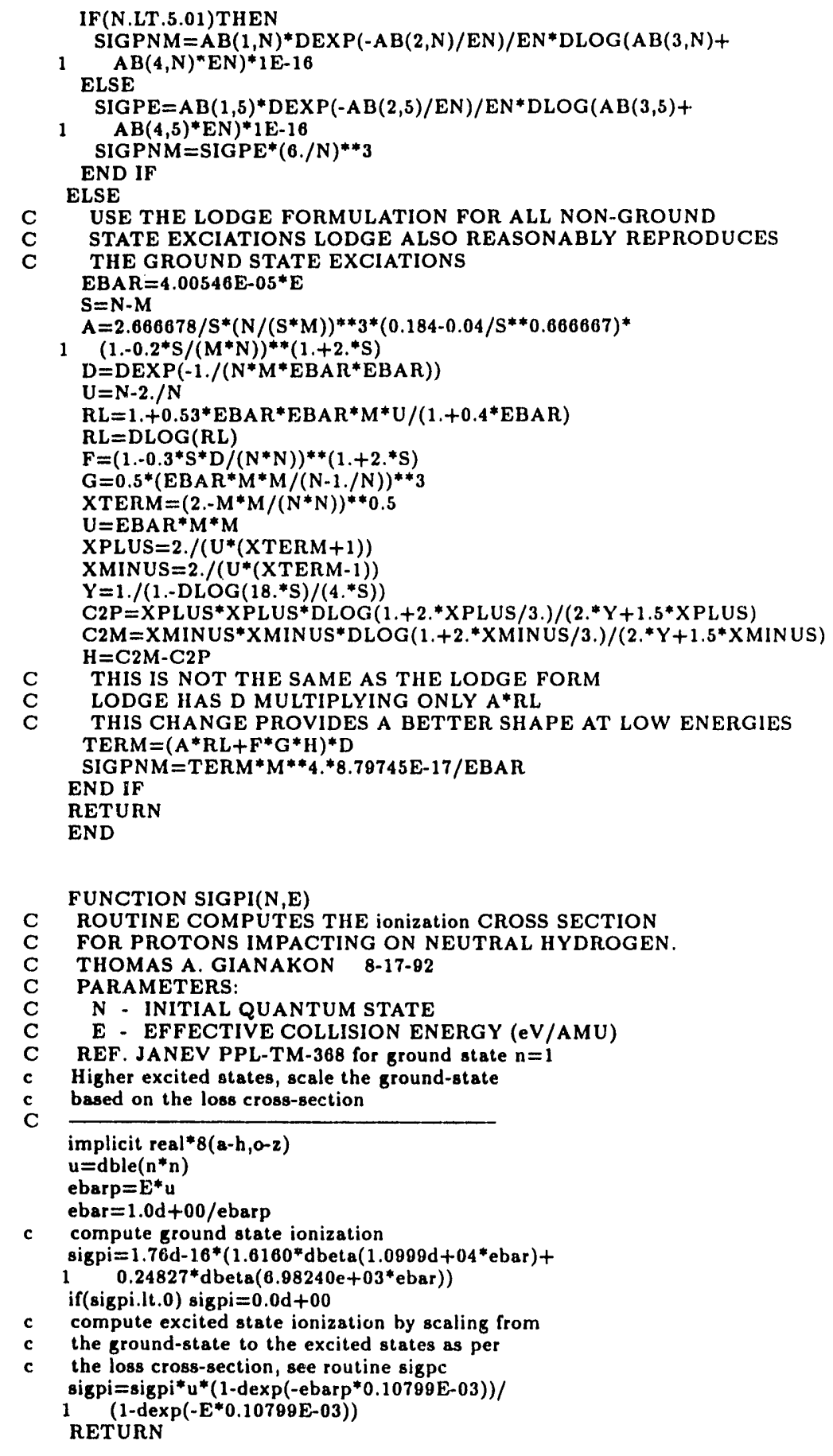


END

FUNCTION SIGPC(N,E)

Routine computes the charge exchange cross-section

for protons impacting on neutral hydrozen by

computing the elctron loss cross-section and

subtracting the ionization cross-section.

THOMAS A. GIANAKON 2-23-82

PARAMETERS:

N - INITIAL QUANTUM STATE

C E - EFFECTIVE COLLISION ENERGY (eV/AMU)

implicit rea]*8(a-h,o-z)

$\mathrm{U}=\mathbf{N} * \mathbf{N}$

c compute the loss cross-section

sigpl $=1.467 \mathrm{E}-11^{*} U^{*}\left(1 .-\operatorname{DEXP}\left(-U^{*} E^{*} 0.10799 \mathrm{E}-03\right)\right) / \mathrm{E}$

c compute the charge exchange cross-section

SIGPC $=$ sigpl-sigpi $(n, e)$

if(sigpc.lt.0) sigpc $=0.0 \mathrm{~d}+00$

RETURN

END

function dbeta( $x)$

Routine computes the function $D$ 'beta used in

the ionization cross-section

Reference Janev and Presnyakov (1980)

implicit real*8(a-h,o-2)

dimension dfen (17)

data dfen $/ 0.057,0.094,0.139,0.187,0.264,0.350,0.401$

$1 \quad 0.387,0.318,0.243,0.186,0.146,0.110,0.0853$

$2 \quad 0.0624,0.0442,0.028$

data ten025/1.778279d+00/

if $(x .1 t .1 .0 d-03)$ then

dbet $a=4.00 d+00 * x * d \log (1.4 d+00 / x)$

elseif(x.gt.10.d+00)then

dbet $a=0.5 d+00^{*} x * \exp \left(-\left(2.0 d+00^{*} x\right) * * 0.5 d+00\right)$

else

$x 0=1 . d+00 / x$

$n=5+4 * d \log 10(x 0)$

$x n=\operatorname{ten} 025^{* *}(n-5)$

1

dbet $a=d f c n(n)+(x 0-x n) *(d f c n(n)-d f c n(n+1))$

end if

$/(x n *(1.0 d+00-\operatorname{ten} 025))$

return

end 


\section{Radiative Decay Rate Code rrate.f}

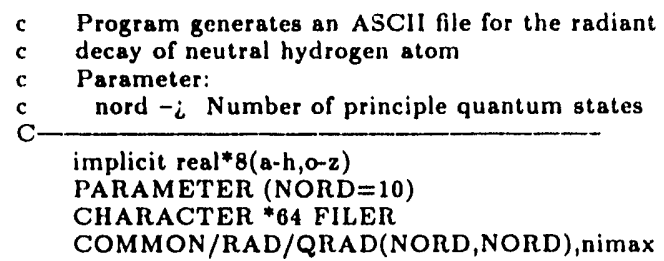

C- COMMON/RAD/QRAD(NORD,NORD), nim

WRITE(*,*)'INPUT THE MAXIMUM NUMBER OF STATES'

READ $(5, *)$ NIMAX

WRITE $\left(*{ }^{*}\right)$ 'INPUT FILENAME FOR RADIANT DECAY'

READ $(*, 8909)$ FILER

C IF(NIMAX.NE.1)THEN

C COMPUTE THE RADIATIVE DECAY

DO $50 \mathrm{~N}=2$, NIMAX

DO $45 \mathrm{M}=1, \mathrm{~N}-1$

$45 \quad \operatorname{QRAD}(N, M)=\operatorname{RADIAT}(M, N)$

50 CONTINUE

END IF

C DUMP DATA

CALL OUTR(FILER)

9990 FORMAT(A)

END






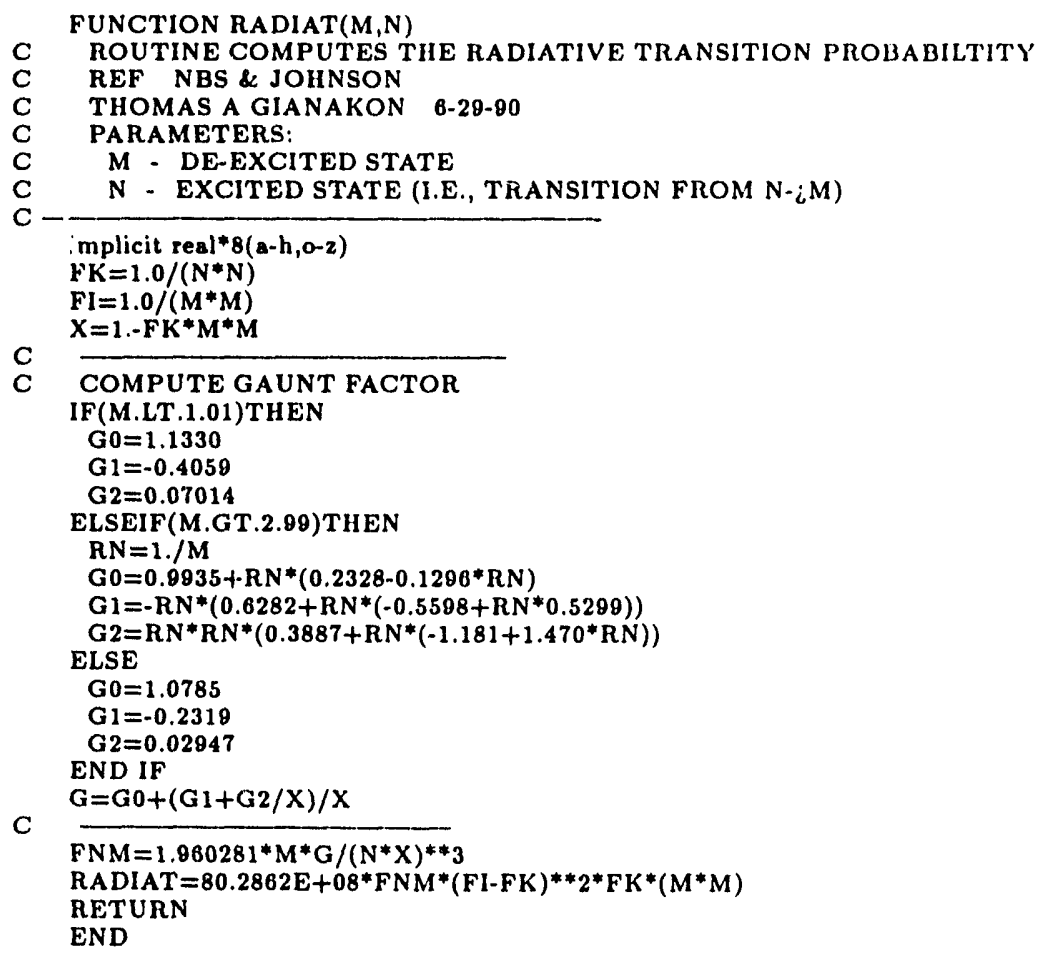




\section{Beam Deposition Code exc_dep.f}

subroutine exc'dep(rtol,atol,zon'dist, state int, ierr)

routine takes zonal temperature and density

for each plasma species of interest and computes

ionization and charge exchange rates for each plasma

species. Calculation is based on excited states of the

neutral beam. The reaction rates are returned in the

common block / fficx / and are normalized to a comparable

ground state calculation.

the following common blocks must be initialized prior

to a call to this routine

(see the include file exc'dep.lis)

/gquad/ - contains gaussian quadrature and weights

/trate/ - reaction rate tables - a call to QREAD

/tspeci/ - parameters which describe the zone plasma

species, beam characteristics and calculational control parameters

\section{parameters:}

$r$ tol - relative tolerance of equation solver (input)

atol - absolute tolerance of equation solver (input)

zon dist - distance $(\mathrm{cm})$ of beam across zone (input)

state int() - on input initial intensities of beam states

ierr - on output intensities

ierr $=0^{-} \quad$; succesful calculation

ierr ; -200 ; leading value gives lsode error

sec the subroutine lsode

-200 ; ierr i 200; table extrapolation problems see the subroutine MRATE

include 'exc'dep.lis'

dimension xg'loc(0:nquad +1), wg' loc $(0: n q u a d+1)$

dimension quad'state( 0 :nquad +1 , nord), zone sum(nord)

dimension state int(nord)

dimension gsii(numspc),gscx (numspc)

dimension rwork(lrw), iwork(liw)

external exc'fcn

c set Lorentz ionization limit

dumb $=7 . d+00 *(3.0 d+00 / b$ perp $) * 0.25 d+00 *$

dumb $=7 . d+00 *(3.0 d+00 / b$
1

nlmax =int (dumb)

if(nlmax.gt.nimax)nlmax =nimax

if(nlmax.lt.1)nlmax $=1$

c set zone quadrature locations and weights

delta $=0.5^{*}$ zon dist

$x g^{\prime} \operatorname{loc}(0)=0.0$

do $10 \mathrm{i}=1$, ngmax

$w g \cdot l o c(i)=$ delta* wgquad $(i)$

$10 \times g \cdot \operatorname{loc}(i)=(1.0 \mathrm{~d}+00+\times \operatorname{gquad}(i)) *$ delta

$x g^{\prime} \operatorname{loc}(n g \max +1)=20 n^{\prime}$ dist

C

c compute reaction rate matrices for each species

call mrate(ierr)

if(ierr.ne.0)return

c mix reaction rate matrices into total reaction rate call qrate(sigma'gs,vo'beam)

c compute total number of particles initially in beam beam dens $=0.0 \mathrm{~d}+00$

do $4 \mathrm{n}=1$, nlmax

4 beam dens=bearn dens, state int( $n)$ 
c populations above Lorentz limit are ionized and

c appear as an ionization value in ffii $(0)$

$\operatorname{fin}(0)=0$

do $5 \mathrm{n}=\mathrm{nlmax}+1$, nimax

c and treat Lorentz ionization limit as species 0

ffi $(0)=$ ffii $(0)+$ state int $(n)$

c add beam density above Lorentz limit to gs calc

beam dens $=$ beam dens + state int $(n)$

5 state int $(n)=0.0 d+00$

C integrate differential equation across zone

itol $=1$

itask $=1$

iopt $=1$

c iwork(6) number of internal calls within isode

iwork $(6)=\operatorname{nimax} * 1000$

$\mathrm{mf}=10$

$x 0=0.0 d+00$

do $20 \mathrm{i}=1$, ngmax +1

istate $=1$

$x 1=x g^{\prime} \operatorname{loc}(i)$

call loode(exc'fcn, nlmax, state int, $x 0, x 1$, itol, rtol,

1 atol,itask, istate,iopt,rwork, Irw, iwork,

2 liw,jac,mf)

if(istate.It.0)then

ierr $=10 *$ istate

when istate $=-1$ excessive work

more than nimax 1000 internal calls

but lsode doesn't integrate to its current endpoint

typically occurs only for large number of states

if(istate.It.-1)then

c true error with lsode

ierr $=100$ *istate

return

end if

c loop to integrate to endpoint on istate $=-1$

c this slows down the calculation by several orders ierr=ierr+iwork(11)

70 istate $=2$

call lsode(exc fen, nlmax, state int, $x 0, \times 1$, itol, rtol,

1 atol,itask, istate,iopt,rwork, Irw,iwork,

2 liw,jac, mf)

if(istate.1t.0)goto 70

end if

c assign values of state intensities to quadrature array

do $15 \mathrm{n}=1, \mathrm{n} / \max$

15 quad'state $(i, n)=$ state int $(n)$

c states above Lorentz limit are set to zero

do $16 \mathrm{n}=\mathrm{nlmax}+1$, nimax

quad'state $(i, n)=0.0 d+00$

20 continue

c perform zone quadrature for each state

do $30 n=1$, nimax

zone $\operatorname{sum}(n)=0.0$

do $25 \mathrm{i}=1$,ngmax

zone'sum $(n)=$ zone sum $(n)+$ quad'state $(i, n) * w g \cdot \operatorname{loc}(i)$

30 continue

c compute zonal deposition based on ground state

term $=$ dexp(-sigma gs*zon dist)

$\mathrm{val}=$ beam $\operatorname{dens}^{*}(1 . d+00$-term $) /($ sigma gs* v0'beam $)$

do $142 \mathrm{i}=1$, nspec

frii $(i)=0.0$

$f \operatorname{cx}(i)=0.0$

$\operatorname{gsii}(i)=q i(i, 1) * \mathrm{val}$ 


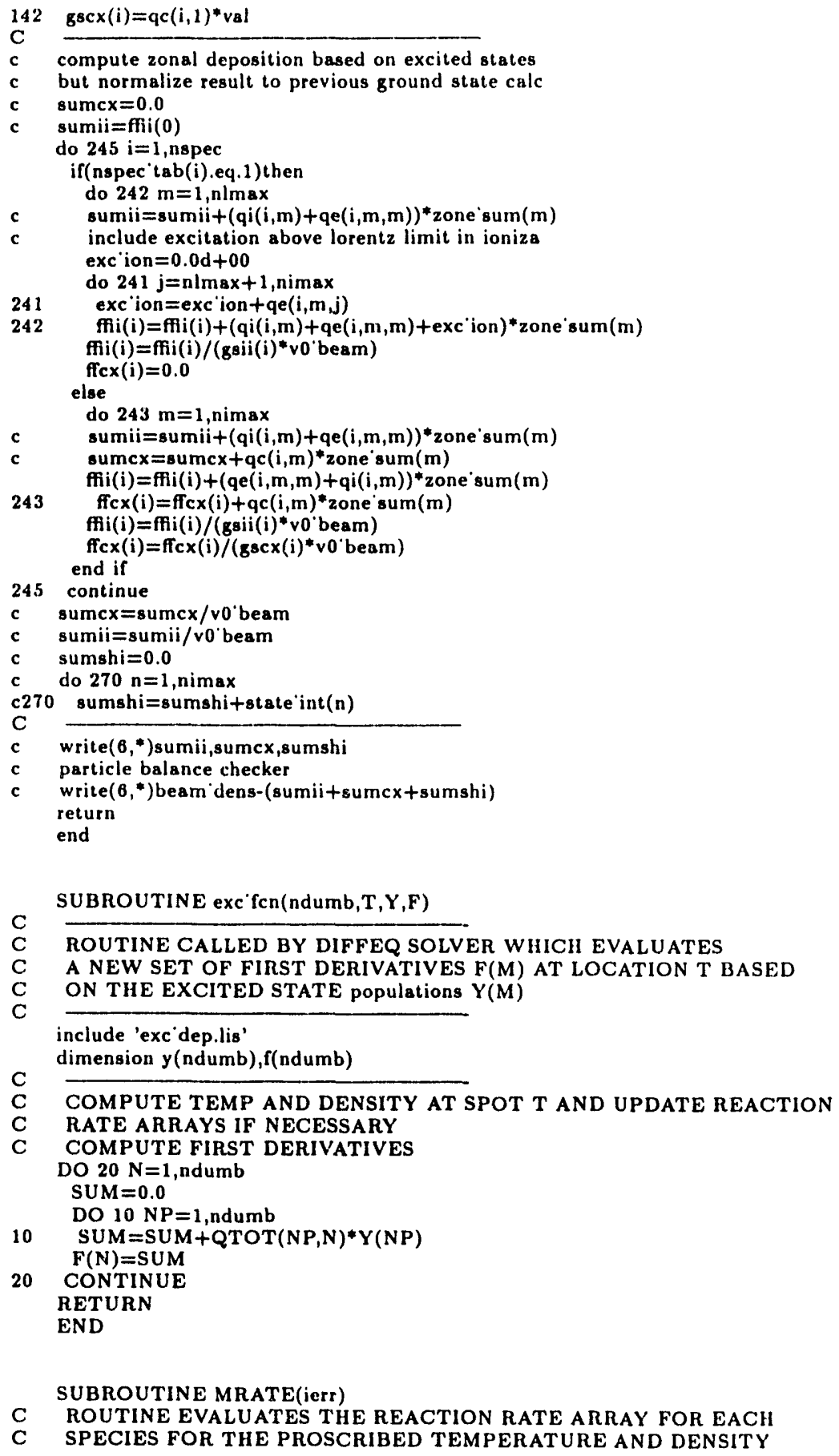




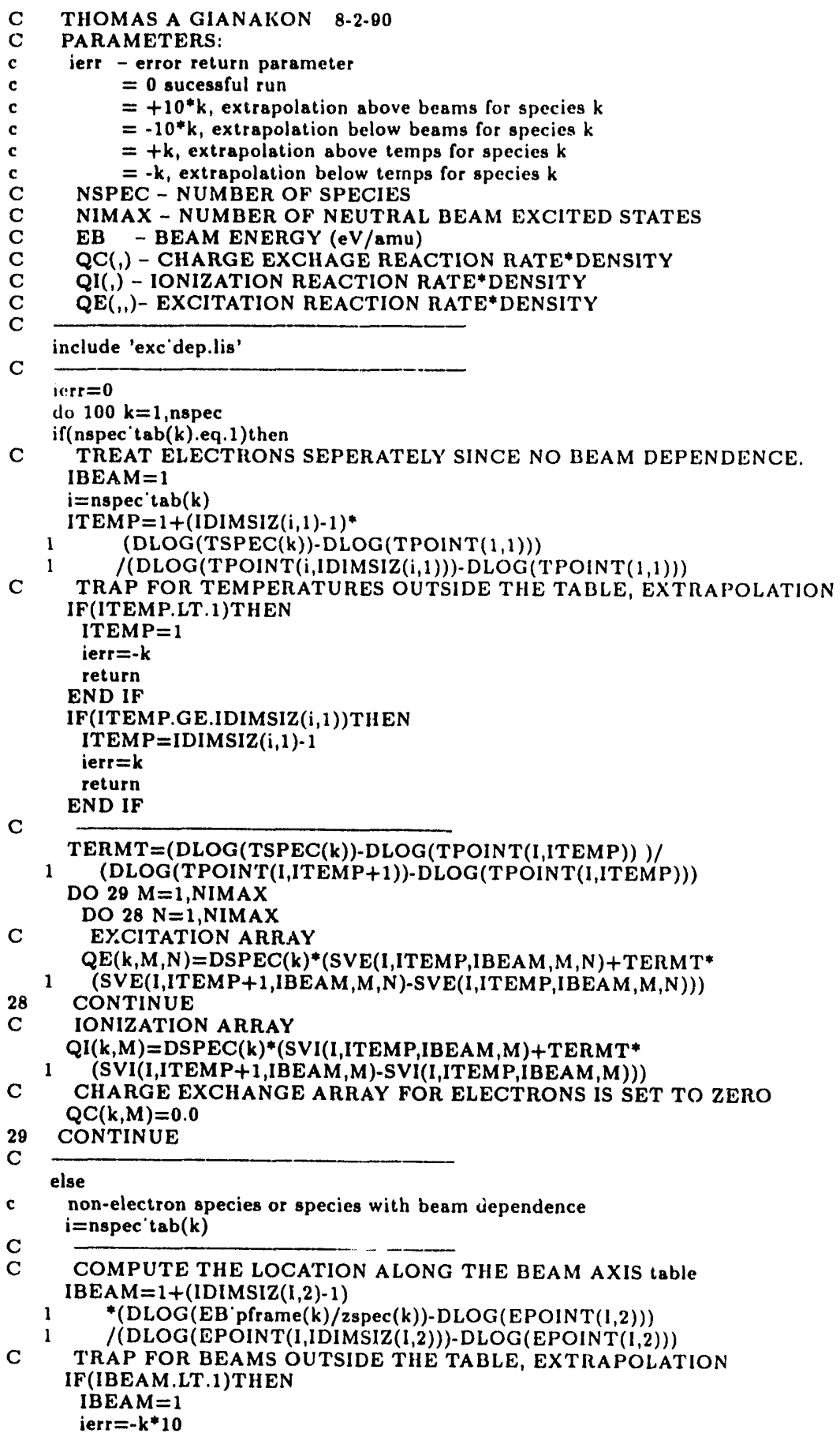




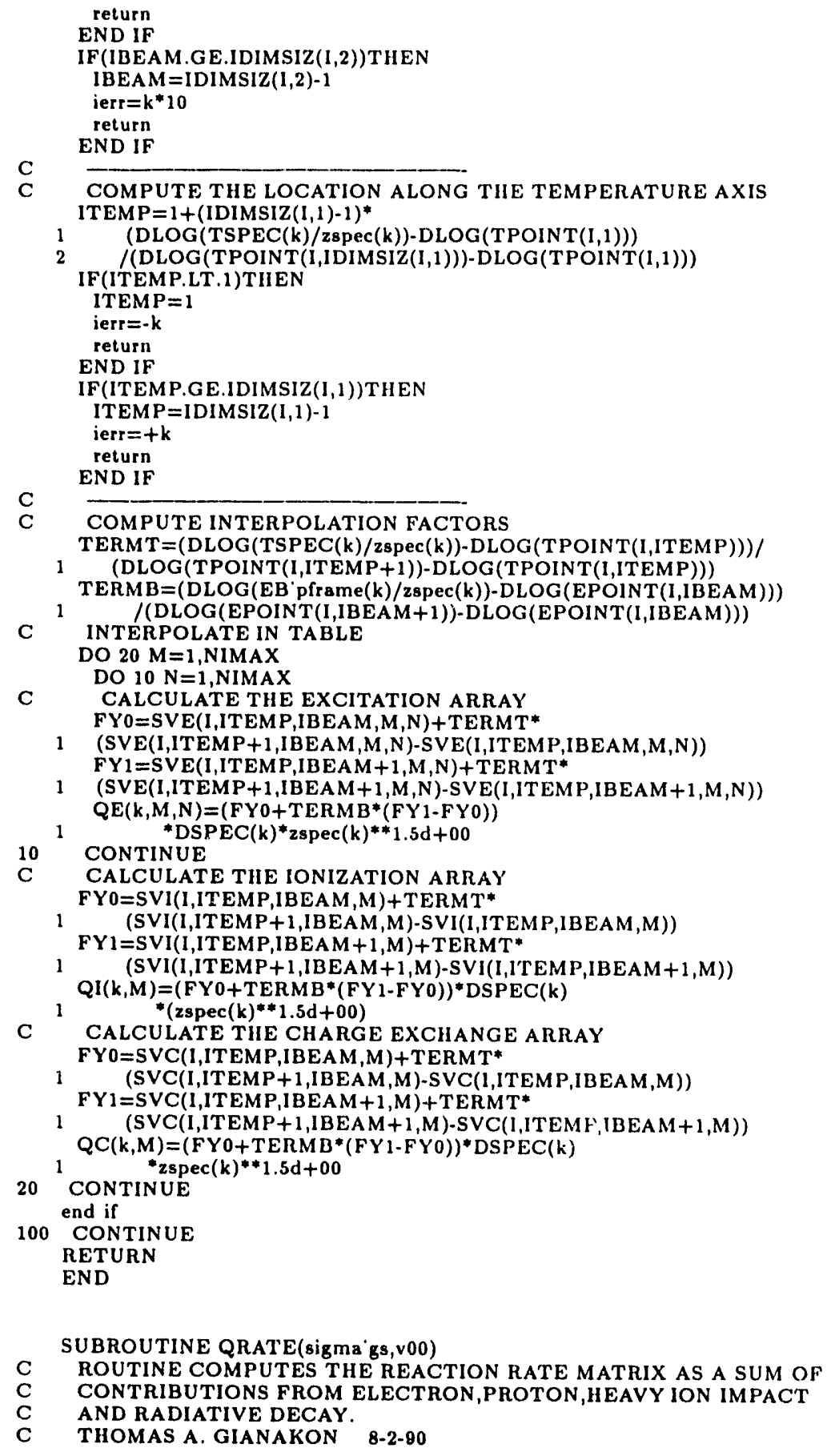




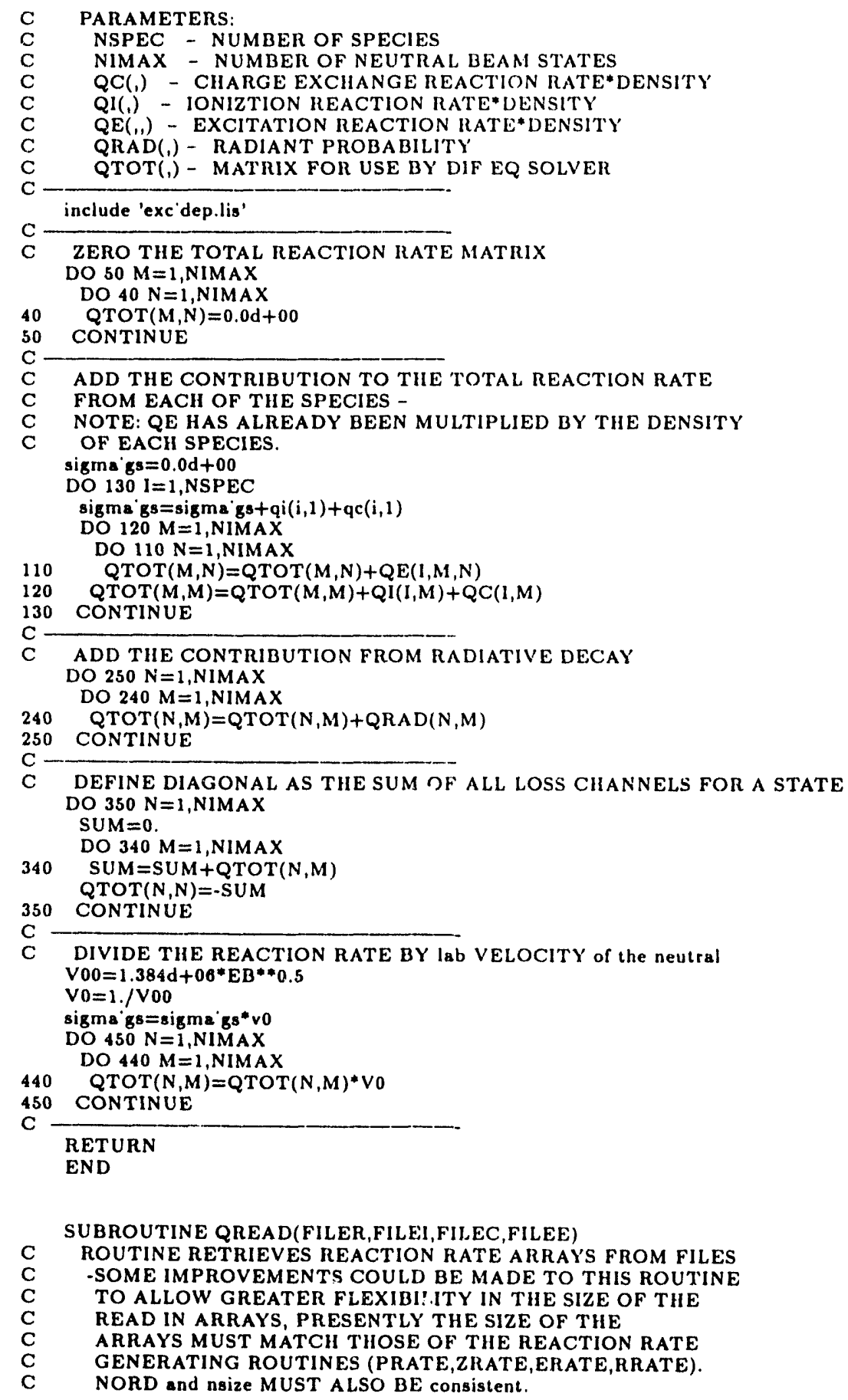




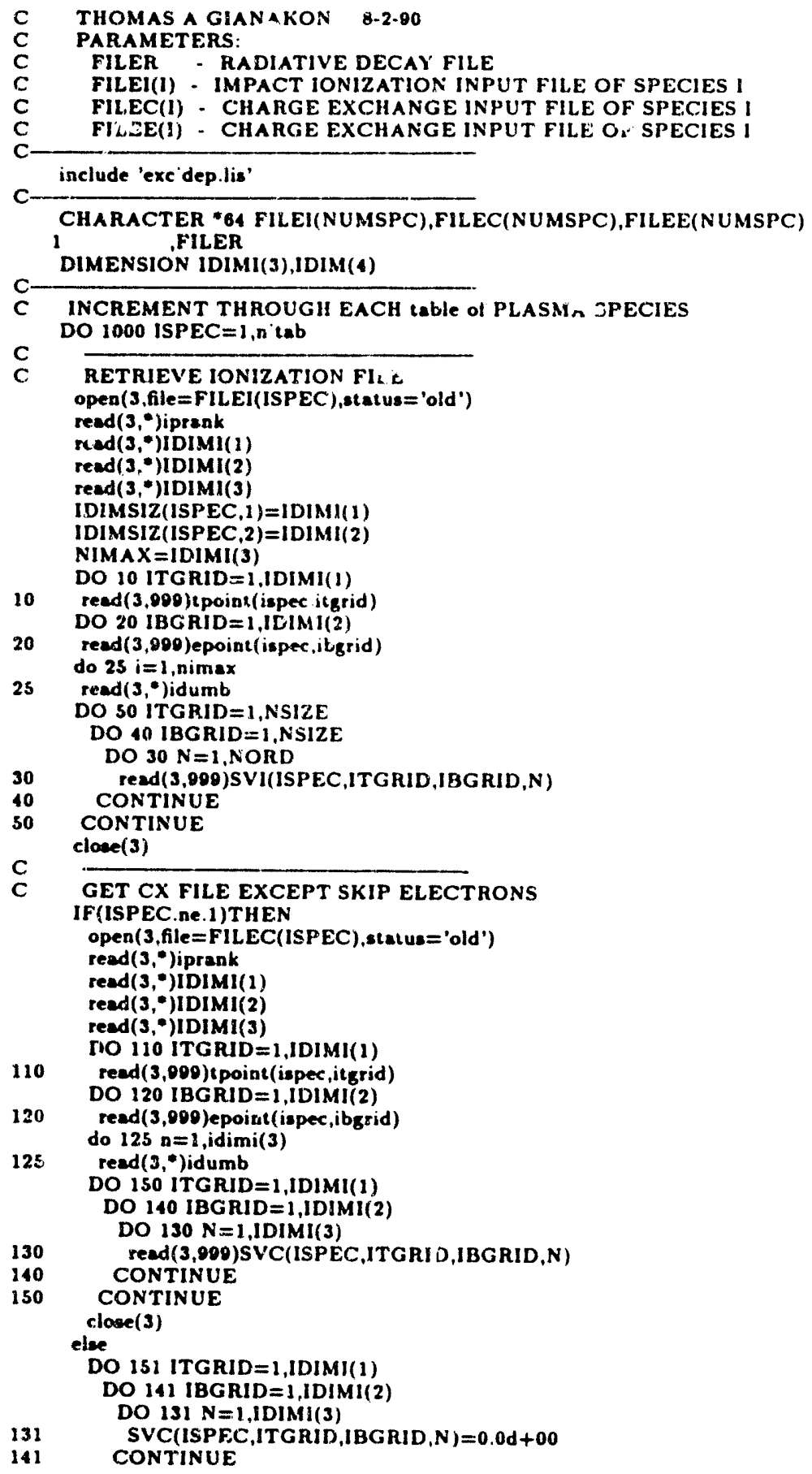




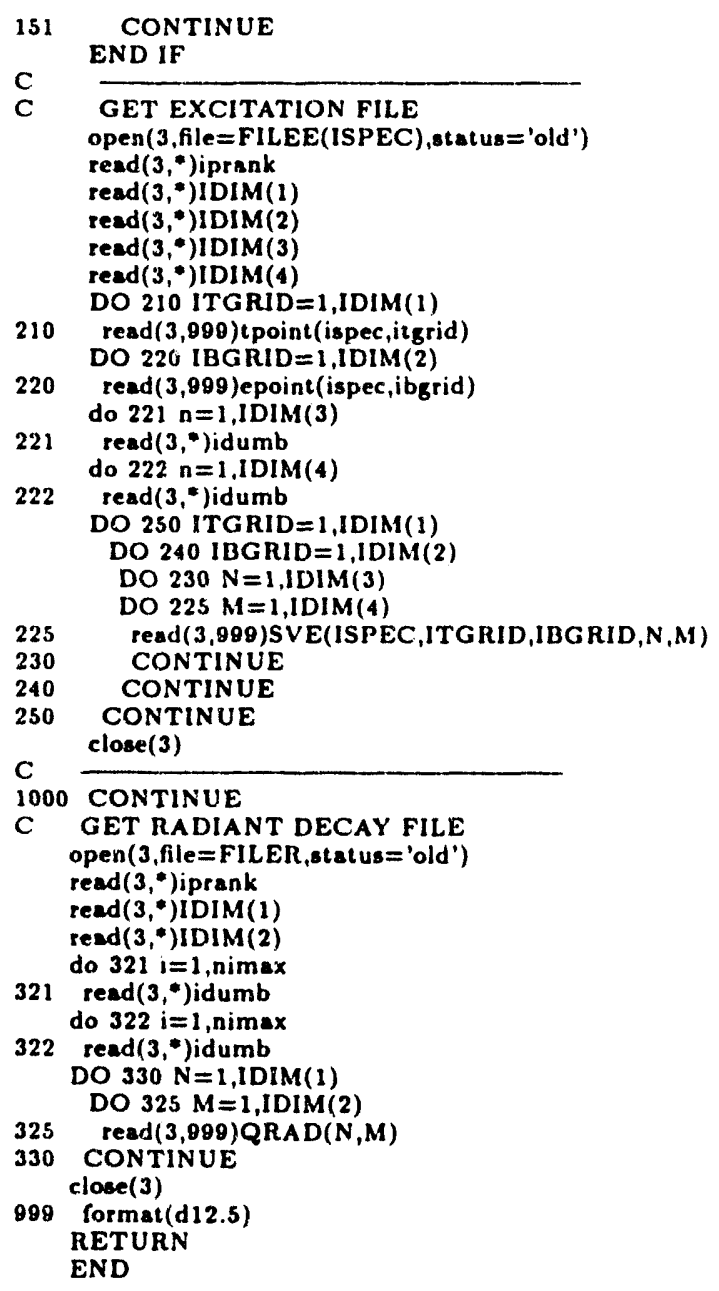




\section{E Beam Deposition Common Block}

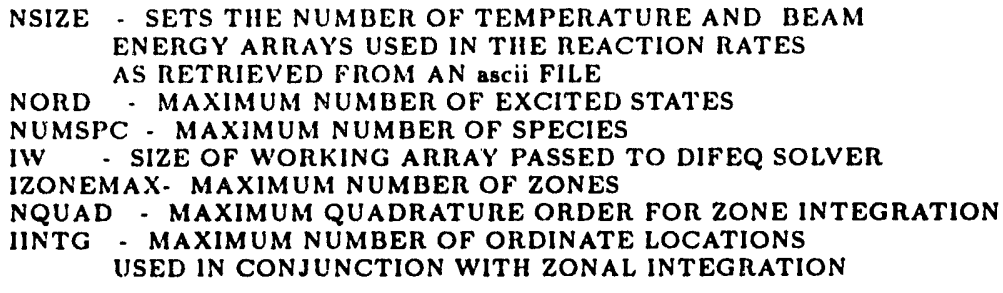




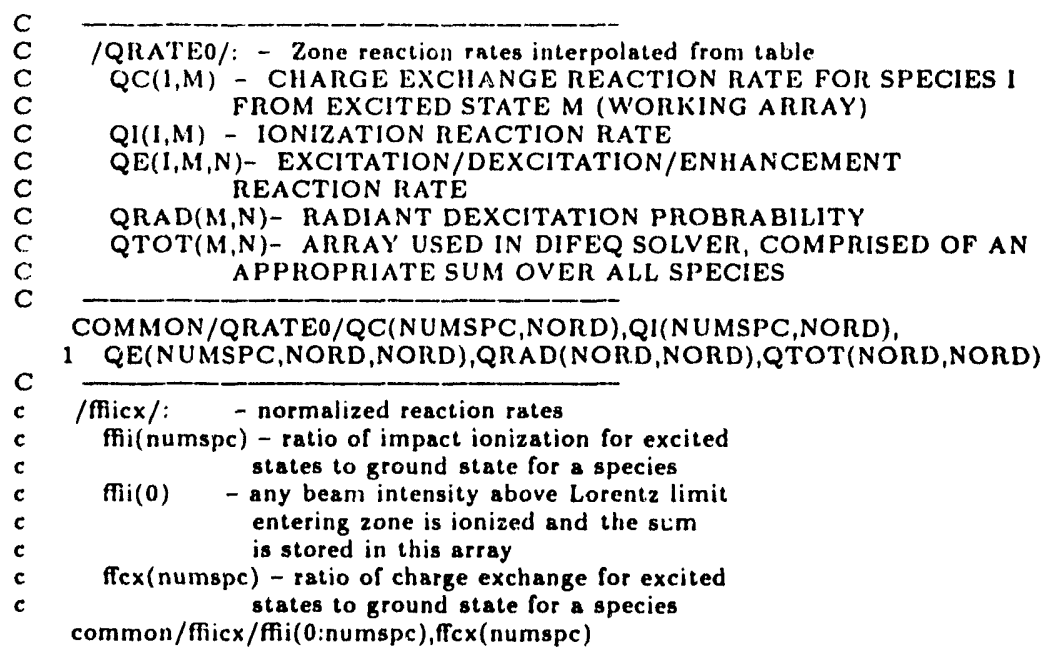



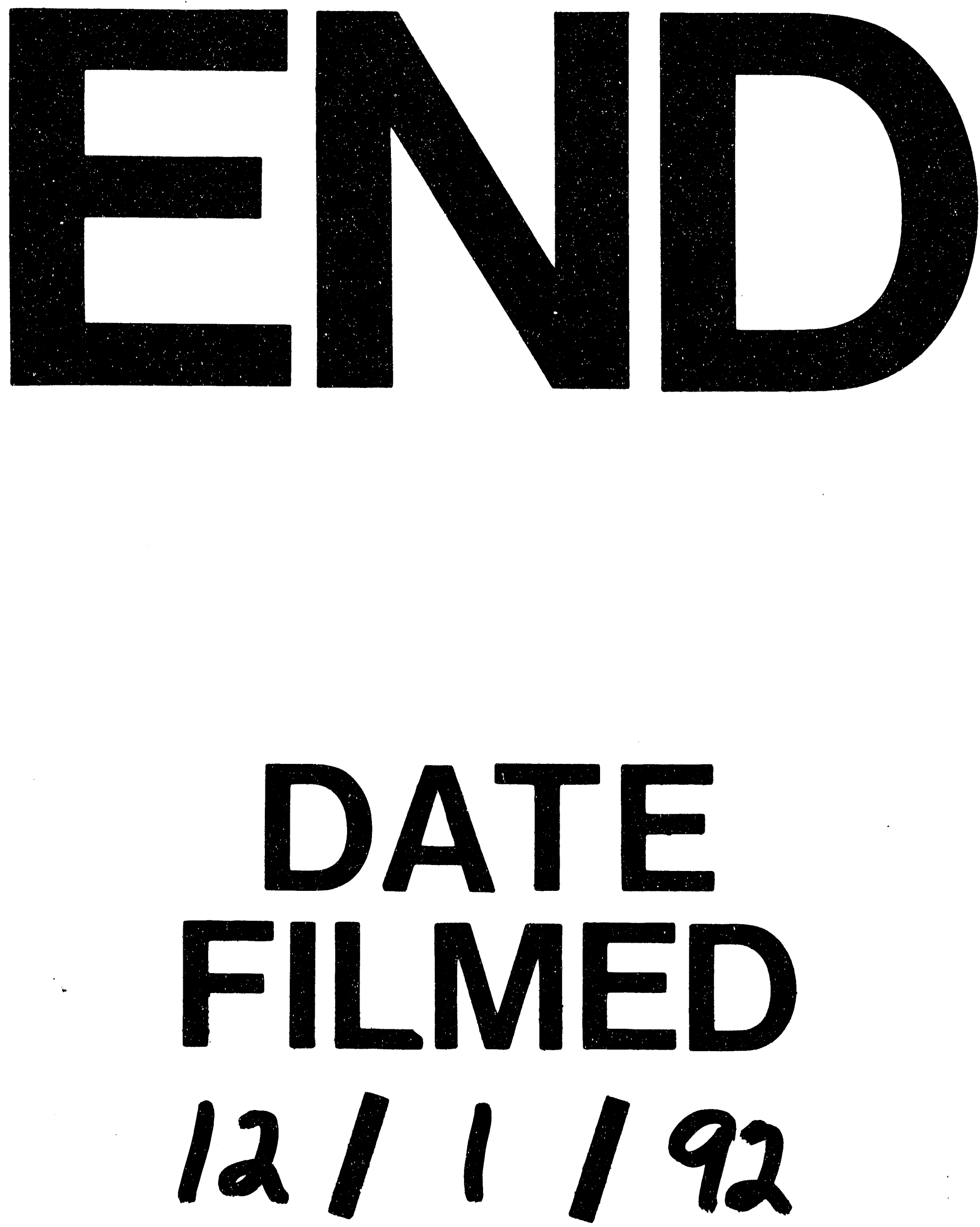


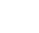

